TRANSACTIONS OF THE

AMERICAN MATHEMATICAL SOCIETY

Volume 359, Number 11, November 2007, Pages 5449-5483

S 0002-9947(07)04194-3

Article electronically published on June 13, 2007

\title{
QUADRATIC HARNESSES, $q$-COMMUTATIONS, AND ORTHOGONAL MARTINGALE POLYNOMIALS
}

\author{
WŁODZIMIERZ BRYC, WOJCIECH MATYSIAK, AND JACEK WESOŁOWSKI
}

\begin{abstract}
We introduce the quadratic harness condition and show that integrable quadratic harnesses have orthogonal martingale polynomials with a three step recurrence that satisfies a $q$-commutation relation. This implies that quadratic harnesses are essentially determined uniquely by five numerical constants. Explicit recurrences for the orthogonal martingale polynomials are derived in several cases of interest.
\end{abstract}

\section{INTRODUCTION}

Hammersley [23] introduced harnesses on $\mathbb{R}^{n}$ as probabilistic models of longrange misorientation in the crystalline structure of metals. Several authors studied mathematical aspects of the concept: Mansuy and Yor [28] analyzed harnesses on $\mathbb{R}_{+}$, Williams [40] analyzed harnesses with the discrete index set; see also [17, 30], [41, [42. The class of random fields on $\mathbb{R}_{+}$which we call quadratic harnesses is related to Hammersley's harnesses in parallel to the relation between martingale and quadratic martingale conditions. Such processes have already been studied implicitly by several authors; see the paragraph following Definition 2.2. In particular, ref. 14 gives a construction of the three parameter family of Markov processes with the quadratic harness property. Some of these processes are related to the free Lévy processes, and some correspond to the non-commutative $q$-Gaussian processes introduced by Frisch and Bourret 21] and studied in 7]. Examples of quadratic harnesses appear in mathematical literature; see [14, 39], and see also more recent works [10, 12, 13. In full generality however, constructions of quadratic harnesses on $\mathbb{R}_{+}$are not yet completely understood. In this paper we concentrate on properties and uniqueness of quadratic harnesses. We show that quadratic harnesses are described by five numerical constants, which, under appropriate integrability conditions, determine the process. We also study related integrability properties of a slightly wider class of processes, improving earlier results of that type [9, Corollary 4], [11, Theorem 2], [39, Theorem 2(1 $\left.{ }^{\circ}\right)$. We show that martingale polynomials associated with a quadratic harness that has finite moments of all orders lead to a $q$-commutation equation

$$
[\mathrm{x}, \mathrm{y}]_{q}=\mathrm{I}+\tau \mathrm{x}^{2}+\sigma \mathrm{y}^{2}+\theta \mathrm{x}+\eta \mathrm{y},
$$

Received by the editors June 8, 2005 and, in revised form, September 26, 2005.

2000 Mathematics Subject Classification. Primary 60J25; Secondary 46L53.

Key words and phrases. Quadratic conditional variances, harnesses, orthogonal martingale polynomials, hypergeometric orthogonal polynomials.

This research was partially supported by NSF grants \#INT-0332062, \#DMS-0504198, and by the C.P. Taft Memorial Fund.

(C)2007 American Mathematical Society 
where $\mathrm{x}, \mathrm{y}$ are infinite matrices satisfying $\mathrm{x} e_{0}=0, \mathrm{y} e_{0}=e_{0}$ with $e_{0}=(1,0, \ldots)^{T}$,

$$
[\mathrm{x}, \mathrm{y}]_{q}=\mathrm{xy}-q \mathrm{yx}
$$

and where $q, \eta, \theta, \sigma, \tau$ are numerical constants that describe the quadratic harness. This hints at more connections with non-commutative probability theory, $q$-Fock space constructions, and classical versions of non-commutative processes; see 7]. Relation (1.1) defines an algebra on two generators. Such algebras have been studied by other authors and can take many equivalent forms; see [38. In this paper we use (1.1) to prove that quadratic harnesses with finite moments of all orders have orthogonal martingale polynomials and to derive their three term recurrences. The explicit three term recurrences that are associated with quadratic harnesses include a four-parameter family of the polynomials in Section 4.2 and a four-parameter family of $q$-orthogonal polynomials in Section 4.3. Later, in 10 we extend the construction from [12 to the construction of a quadratic harness, which we call the bi-Poisson process, based on one of the explicit recurrences from this paper.

Some of our arguments rely on the symmetries of the problem, though perhaps we did not explore the symmetries deeply enough. The family of quadratic harnesses that we study is invariant under the action of the translations and reflections of $\mathbb{R}$, and the associated affine Hecke algebra is known to be associated with the AskeyWilson polynomials; see [29]. It is plausible that the theory of affine root systems 27. might lead to additional progress.

The paper is organized as follows. In Section 2 we define quadratic harnesses and state the main results. Proofs of the main results are given in Section 3 . with some more technical proofs deferred to the appendix. In Section 4 we derive explicit recurrences for the orthogonal martingale polynomials. We also introduce an operator technique motivated by umbral calculus that simplifies the proof of the quadratic harness property.

Note added after submission. After this paper was submitted, we learned that equations similar to (1.1) appear in the analysis of the asymmetric exclusion process [16, and that various tri-diagonal matrix solutions are known; see in particular 36, Section 4], where such solutions are elegantly expressed in terms of the Jacobi matrices of the Askey-Wilson polynomials with appropriate parameters. Essler and Rittenberg [19] consider the general quadratic $q$-commutation relation (1.1) and provide details in two important cases when $\eta=\theta=0$, or $\eta=\theta=1$.

\section{Definitions AND MAIN RESUlts}

2.1. Harnesses and quadratic harnesses. Let $\left(X_{t}\right)_{t>0}$ be a separable square integrable stochastic process with $\sigma$-fields $\mathcal{F}_{s, u}=\sigma\left\{X_{t}: t \in(0, s] \cup[u, \infty)\right\}$. Consider the following two "increment" functionals:

$$
\begin{aligned}
\Delta_{t, u}=\Delta_{t, u}(X) & =\frac{X_{u}-X_{t}}{u-t}, \\
\widetilde{\Delta}_{t, u}=\widetilde{\Delta}_{t, u}(X) & =\frac{u X_{t}-t X_{u}}{u-t} .
\end{aligned}
$$

Both functionals appear in the statement of Theorem 2.2 below, and the second one is the time-inverse of the first one. Namely, if $\left(\tilde{X}_{t}\right)_{t>0}$ denotes the time-inverse process $\left(t X_{1 / t}\right)_{t>0}$, then

$$
\widetilde{\Delta}_{t, u}(X)=\Delta_{1 / u, 1 / t}(\widetilde{X}), 0<t<u .
$$


Definition $2.1(28])$. An integrable process $\left(X_{t}\right)_{t>0}$ is a (simple) harness if

$$
\mathbb{E}\left[\Delta_{s, t} \mid \mathcal{F}_{r, u}\right]=\Delta_{r, u}
$$

for every $r<s<t<u$.

A trivial example of a harness is a Gaussian process with covariance (2.5); additional examples follow Definition 2.2. As pointed out in [28, the harness condition is equivalent to the linearity of the regression property

$$
\mathbb{E}\left[X_{t} \mid \mathcal{F}_{s, u}\right]=a_{t, s, u} X_{s}+b_{t, s, u} X_{u},
$$

where the coefficients are given by

$$
a_{t, s, u}=\frac{u-t}{u-s}, \quad b_{t, s, u}=\frac{t-s}{u-s} .
$$

It is clear that $a_{t, s, u}+b_{t, s, u}=1$ and $s a_{t, s, u}+u b_{t, s, u}=t$; these two identities will often be used. Since condition (2.3) is invariant under the time-inversion $\left(X_{t}\right) \mapsto\left(t X_{1 / t}\right)$, another equivalent condition for a harness is

$$
\mathbb{E}\left[\widetilde{\Delta}_{s, t} \mid \mathcal{F}_{r, u}\right]=\widetilde{\Delta}_{r, u}, r<s<t<u .
$$

The general form of the covariance of a square-integrable harness is as follows.

Proposition 2.1. If $\left(X_{t}\right)$ is a square-integrable centered harness on $\mathbb{R}_{+}$, then there are constants $c_{0}, c_{1}, c_{2}, c_{3}$ such that for $s \leq t$ we have

$$
\mathbb{E}\left(X_{t} X_{s}\right)=c_{0}+c_{1} s+c_{2} t+c_{3} s t .
$$

Proof. Multiplying (2.3) by $X_{s}$ and averaging we get $\mathbb{E}\left(X_{s} X_{t}\right)=a_{t, s, u} \mathbb{E}\left(X_{s}^{2}\right)+$ $b_{t, s, u} \mathbb{E}\left(X_{s} X_{u}\right)$. Thus $\mathbb{E}\left(X_{s} X_{t}\right)=\alpha(s)+\beta(s) t$ is linear in $t$ and $\mathbb{E}\left(X_{t} X_{u}\right)=$ $a_{t, s, u} \mathbb{E}\left(X_{s} X_{u}\right)+b_{t, s, u} \mathbb{E}\left(X_{u}^{2}\right)=\gamma(u)+\delta(u) t$ is linear in $t$. This gives a functional equation

$$
\alpha(s)+\beta(s) t=\gamma(t)+\delta(t) s,
$$

which, by taking $s=0$, gives $\gamma(t)=\alpha(0)+\beta(0) t$. Thus $\mathbb{E}\left(X_{s} X_{t}\right)=c_{0}+c_{1} s+c_{2} t+$ $c_{3} s t$.

For a centered standardized square-integrable $Z$ that is independent of a quadratic harness $\left(X_{t}\right)$, taking $\left(\tilde{X}_{t}\right)=\left(X_{t}+(t+1) Z\right)$ we get a quadratic harness with $\mathbb{E}\left(\tilde{X}_{s} \tilde{X}_{t}\right)=\mathbb{E}\left(X_{s} X_{t}\right)+1+t+s+t s$. To avoid such non-uniqueness, throughout this paper we assume that

$$
\mathbb{E}\left(X_{t}\right)=0, \mathbb{E}\left(X_{t} X_{s}\right)=\min \{t, s\} .
$$

If (2.6) and (2.3) hold true and $0<s \leq t \leq u$, then

$$
\mathbb{E}\left[X_{t} \mid \mathcal{F}_{\geq u}\right]=\frac{t}{u} X_{u}
$$

and

$$
\mathbb{E}\left[X_{t} \mid \mathcal{F}_{\leq s}\right]=X_{s}
$$

see [14, (4) and (5)]. From the martingale (2.8) and reverse martingale (2.7) conditions it follows that the limits $X_{0}:=\lim _{t \backslash 0} X_{t}$, and $\lim _{t \rightarrow \infty} X_{t} / t$ exist with probability one. Under assumption (2.6) we have

$$
\lim _{t \searrow 0} X_{t}=0, \lim _{t \rightarrow \infty} X_{t} / t=0,
$$


so without loss of generality we may extend $\left(X_{t}\right)_{t>0}$ to include the value $X_{0}=0$ when convenient. Similarly, we may extend $\left(\widetilde{X}_{t}\right)_{t>0}=\left(t X_{1 / t}\right)_{t>0}$ to include the value $\widetilde{X}_{0}=0$ corresponding to $t=0$.

We now turn to the quadratic harness condition. The familiar quadratic martingale condition associated with the martingale property (2.8) can be written as

$$
\mathbb{E}\left[X_{t}^{2} \mid \mathcal{F}_{\leq s}\right]=X_{s}^{2}+t-s .
$$

This suggests that the quadratic harness condition associated with the simple harness property (2.3) should be written as

$$
\mathbb{E}\left[X_{t}^{2} \mid \mathcal{F}_{s, u}\right]=Q_{t, s, u}\left(X_{s}, X_{u}\right),
$$

where

$$
Q_{t, s, u}(x, y)=A_{t, s, u} x^{2}+B_{t, s, u} x y+C_{t, s, u} y^{2}+D_{t, s, u} x+E_{t, s, u} y+F_{t, s, u}
$$

is a quadratic form in variables $x, y$ with time-dependent coefficients; under assumption (2.6) we trivially have

$$
s A_{t, s, u}+s B_{t, s, u}+u C_{t, s, u}+F_{t, s, u}=t .
$$

Definition 2.2. A square integrable process $\left(X_{t}\right)_{t>0}$ is a quadratic harness on $\mathbb{R}_{+}$ if it satisfies conditions (2.3) and (2.11).

The well-known examples of quadratic harnesses are the Wiener, Poisson, and Gamma processes. Ref. 39 identifies all quadratic harnesses with covariance (2.6) when the quadratic form on the right hand side of (2.11) is such that the corresponding conditional variance is a function of the increments $X_{u}-X_{s}$ only; this adds to the already listed examples two additional Lévy processes: the Pascal, and Meixner processes. A related non-commutative form of condition (2.11) appears in [6. The main result of 14 asserts that quadratic harnesses with covariance (2.6) which satisfy the quadratic martingale condition (2.10) are in fact uniquely determined $q$-Meixner Markov processes. A quadratic harness that does not satisfy condition (2.10) is analyzed in [12.

2.2. Five-parameter representation. Generically, the quadratic form $Q_{t, s, u}$ on the right hand side of (2.11) is determined uniquely up to five numerical parameters.

Theorem 2.2. Let $\left(X_{t}\right)$ be a quadratic harness with covariance (2.6). Suppose that (2.11) holds with $F_{t, s, u} \neq 0$ for all $0<s<t<u$, and that $1, X_{s}, X_{t}, X_{s} X_{t}, X_{s}^{2}, X_{t}^{2}$ are linearly independent for all $0<s<t$. Then there exist $\eta, \theta \in \mathbb{R}, \sigma, \tau \geq 0$, and $q \leq 1+2 \sqrt{\sigma \tau}$ such that

$$
\operatorname{Var}\left[X_{t} \mid \mathcal{F}_{s, u}\right]=F_{t, s, u} K\left(\frac{X_{u}-X_{s}}{u-s}, \frac{u X_{s}-s X_{u}}{u-s}\right)
$$

for all $0<s<t<u$, where

$$
F_{t, s, u}=\frac{(u-t)(t-s)}{u(1+\sigma s)+\tau-q s}
$$

and

$$
K(\mathrm{x}, \mathrm{y})=1+\theta \mathrm{x}+\tau \mathrm{x}^{2}+\eta \mathrm{y}+\sigma \mathrm{y}^{2}-[\mathrm{x}, \mathrm{y}]_{q}
$$

comes from (1.1), and is applied here to commuting variables $\Delta_{s, u}, \widetilde{\Delta}_{s, u}$. 
Recall that the conditional variance of $X$ with respect to a $\sigma$-field $\mathcal{F}$ is defined as $\operatorname{Var}[X \mid \mathcal{F}]=\mathbb{E}\left[X^{2} \mid \mathcal{F}\right]-(\mathbb{E}[X \mid \mathcal{F}])^{2}$.

Remark 2.1. Time-inversion $\left(X_{t}\right) \mapsto\left(t X_{1 / t}\right)$ preserves the class of quadratic harnesses, modifying the coefficients in (2.11). More precisely, suppose $\left(X_{t}\right)$ satisfies the assumptions of Theorem 2.2, and let $\widetilde{X}_{t}=t X_{1 / t}$ be its time inverse. Then $\left(\widetilde{X}_{t}\right)$ is a quadratic harness with respect to its $\sigma$-fields $\widetilde{\mathcal{F}}_{s, u}=\mathcal{F}_{1 / u, 1 / s}$, and (2.14) holds with the roles of the parameters $(\eta, \theta)$ and $(\sigma, \tau)$ switched within each pair:

$$
\operatorname{Var}\left[\widetilde{X}_{t} \mid \widetilde{\mathcal{F}}_{s, u}\right]=\widetilde{F}_{t, s, u} \widetilde{K}\left(\frac{\widetilde{X}_{u}-\widetilde{X}_{s}}{u-s}, \frac{u \widetilde{X}_{s}-s \widetilde{X}_{u}}{u-s}\right)
$$

where $\widetilde{F}_{t, s, u}=\frac{(u-t)(t-s)}{u(1+\tau s)+\sigma-q s}$ and $\widetilde{K}(\mathrm{x}, \mathrm{y})=1+\eta \mathrm{x}+\sigma \mathrm{x}^{2}+\theta \mathrm{y}+\tau \mathrm{y}^{2}-[\mathrm{x}, \mathrm{y}]_{q}$. Ref. 22 gives criteria for time-inversion invariance of Markov processes.

2.3. Orthogonal martingale polynomials. Suppose that a quadratic harness $\left(X_{t}\right)$ has moments of all orders and martingale polynomials $p_{n}(x ; t)$ of all degrees $n \geq 0$, that is,

$$
\mathbb{E}\left[p_{n}\left(X_{t} ; t\right) \mid \mathcal{F}_{\leq s}\right]=p_{n}\left(X_{s} ; s\right), 0<s<t .
$$

Clearly, $p_{0}=1$ and $p_{1}(x ; t)=x$ are natural initial choices; see (2.8). Since $x p_{n}(x ; t)$ is a polynomial of degree $n+1$, it follows that

$$
x p_{n}(x ; t)=\sum_{k=0}^{n+1} C_{k, n}(t) p_{k}(x ; t) .
$$

Theorem 2.3. Suppose a quadratic harness $\left(X_{t}\right)$ with covariance (2.6) and conditional variance (2.14) has finite moments of all orders and martingale polynomials $p_{n}(x ; t)$. If for each $t>0$ the random variable $X_{t}$ has infinite support, then recurrence (2.17) holds with the infinite matrices

$$
\mathbf{C}_{t}:=\left[\begin{array}{llllll}
C_{00}(t) & C_{01}(t) & C_{02}(t) & C_{03}(t) & \ldots & \\
C_{10}(t) & C_{11}(t) & C_{12}(t) & C_{13}(t) & \ldots & \\
0 & C_{21}(t) & C_{22}(t) & C_{23}(t) & \ldots & \\
0 & 0 & C_{32}(t) & C_{33}(t) & \ldots & \\
0 & 0 & 0 & C_{43}(t) & \ddots & \\
\vdots & \vdots & \vdots & & & \ddots
\end{array}\right]
$$

given by

$$
\mathbf{C}_{t}=t \mathrm{x}+\mathrm{y}, t>0
$$

and the infinite matrices $\mathbf{x}=\mathbf{C}_{1}-\mathbf{C}_{0}, \mathbf{y}=\mathbf{C}_{0}$ satisfy equation (1.1).

From Theorem 2.3 we derive a number of equations that eventually determine the orthogonal martingale polynomials. Namely, it is well known that for orthogonal martingale polynomials $\left\{p_{n}(x ; t)\right\}$ recurrence (2.17) holds with a tri-diagonal matrix C; see [15]. Writing (2.17) as

$$
x p_{n}(x ; t)=a_{n}(t) p_{n+1}(x ; t)+b_{n}(t) p_{n}(x ; t)+c_{n}(t) p_{n-1}(x ; t), n \geq 0,
$$


from (2.18) we get

$$
a_{n}(t)=\sigma \alpha_{n+1} t+\beta_{n+1}, \quad b_{n}(t)=\gamma_{n} t+\delta_{n}, \quad c_{n}(t)=\varepsilon_{n} t+\varphi_{n},
$$

and the coefficients in (2.20) satisfy a number of equations that result from the $q$-commutation equation (1.1). Setting $p_{-1}(x ; t)=0, p_{0}(x ; t)=1, p_{1}(x ; t)=x$, and using (2.6) we see that the initial values are given by

$$
\alpha_{1}=0, \beta_{1}=1, \gamma_{0}=\delta_{0}=0, \varepsilon_{1}=1, \varphi_{1}=0 .
$$

For $n \geq 1$, (1.1) implies that coefficients $\alpha_{n}, \beta_{n}, \gamma_{n}, \delta_{n}, \varphi_{n}, \varepsilon_{n}$ satisfy

$$
\begin{aligned}
& \sigma^{2} \tau \alpha_{n} \alpha_{n+1}+\sigma \alpha_{n} \beta_{n+1} q+\sigma \beta_{n} \beta_{n+1}=\sigma \alpha_{n+1} \beta_{n}, \\
& \beta_{n+1} \gamma_{n+1}+\sigma \alpha_{n+1} \delta_{n} \\
& =\sigma \alpha_{n+1}\left(\gamma_{n}+\gamma_{n+1}\right) \tau+\left(\sigma \alpha_{n+1} \delta_{n+1}+\beta_{n+1} \gamma_{n}\right) q+\beta_{n+1}\left(\delta_{n}+\delta_{n+1}\right) \sigma \\
& +\sigma \alpha_{n+1} \theta+\beta_{n+1} \eta \\
& \beta_{n+1} \varepsilon_{n+1}+\gamma_{n} \delta_{n}+\sigma \alpha_{n} \varphi_{n} \\
& =\left(\sigma \alpha_{n+1} \varepsilon_{n+1}+\gamma_{n}^{2}+\sigma \alpha_{n} \varepsilon_{n}\right) \tau+\left(\sigma \alpha_{n+1} \varphi_{n+1}+\gamma_{n} \delta_{n}+\beta_{n} \varepsilon_{n}\right) q \\
& +\left(\beta_{n+1} \varphi_{n+1}+\delta_{n}^{2}+\beta_{n} \varphi_{n}\right) \sigma+\gamma_{n} \theta+\delta_{n} \eta+1, \\
& \gamma_{n-1} \varphi_{n}+\delta_{n} \varepsilon_{n} \\
& =\left(\gamma_{n-1}+\gamma_{n}\right) \varepsilon_{n} \tau+\left(\gamma_{n} \varphi_{n}+\delta_{n-1} \varepsilon_{n}\right) q+\left(\delta_{n-1}+\delta_{n}\right) \varphi_{n} \sigma+\varepsilon_{n} \theta+\varphi_{n} \eta, \\
& \varepsilon_{n} \varphi_{n+1}=\varepsilon_{n} \varepsilon_{n+1} \tau+\varepsilon_{n+1} \varphi_{n} q+\varphi_{n} \varphi_{n+1} \sigma .
\end{aligned}
$$

If $a_{n}(t) \neq 0$ for all $n$, then the three step recurrence (2.19) defines a family of polynomials in the variable $x$. We now show that these are indeed the martingale orthogonal polynomials for $\left(X_{t}\right)$.

Theorem 2.4. Suppose a quadratic harness $\left(X_{t}\right)$ with covariance (2.6) and conditional variance (2.14) has finite moments of all orders, parameters $q, \eta, \theta, \sigma, \tau$ are such that $(q, \sigma \tau) \neq(-1,1)$, and the equations (2.21)-(2.26) have a solution such that $a_{n}(t) \neq 0$ for all $t \geq 0, n \geq 0$. Let $\left\{p_{n}(x ; t): n \geq 0, t>0\right\}$ satisfy (2.19) and (2.20) for $n=0,1, \ldots$ with $p_{-1}(x, t)=0, p_{0}(x, t)=1, p_{1}(x, t)=x$. Then $\left\{p_{n}(x ; t)\right\}$ are orthogonal martingale polynomials for $\left(X_{t}\right)$. (In particular, $a_{n}(t) c_{n}(t) \geq 0$.)

In Section 4, we give sufficient conditions in terms of parameters $q, \eta, \theta, \sigma, \tau$ for the assumptions of Theorem 2.4 to be satisfied, and we derive explicit three step recurrences for the orthogonal martingale polynomials in several cases of interest. If quadratic harnesses $\left(X_{t}\right)_{t>0}$ and $\left(Y_{t}\right)_{t>0}$ satisfy the assumptions of Theorem 2.4 with the same parameters $q, \eta, \theta, \sigma, \tau$, then

$$
\mathbb{E}\left(X_{t_{1}}^{n_{1}} X_{t_{2}}^{n_{2}} \ldots X_{t_{k}}^{n_{k}}\right)=\mathbb{E}\left(Y_{t_{1}}^{n_{1}} Y_{t_{2}}^{n_{2}} \ldots Y_{t_{k}}^{n_{k}}\right)
$$

for all $k \geq 1,0<t_{1}<t_{2}<\cdots<t_{k}$, and $n_{1}, \ldots, n_{k} \in \mathbb{N}$. Under appropriate integrability conditions, this implies that $\left(X_{t}\right)$ is a uniquely determined Markov process with parameters $q, \eta, \theta, \sigma, \tau$. In particular, this is the case when $\sup _{n}\left|b_{n}(t)\right|<\infty$ and $\sup _{n}\left|a_{n-1}(t) c_{n}(t)\right|<\infty$, as in this case recurrence (2.19) corresponds to a compactly supported measure; see [4, Section 2]. However, the question of existence of such a process is non-trivial and the constructions are known only in special cases; see [10, [12, 14]. 
Next, we show that the integrability assumption of Theorem 2.4 is automatically satisfied if $\sigma \tau=0$. We remark that the result stated below does not use the full power of the quadratic harness condition and generalizes [11, Theorem 2] and [39, Theorem $2\left(1^{\circ}\right)$ ]. Since

$$
\operatorname{Var}\left[X_{t} \mid \mathcal{F}_{s, u}\right]=\mathbb{E}\left[X_{t}^{2} \mid \mathcal{F}_{s, u}\right]-\left(a_{t, s, u} X_{s}+b_{t, s, u} X_{u}\right)^{2},
$$

using (2.9) we can pass to the limit in (2.14) as $u \rightarrow \infty$ or as $s \rightarrow 0$. This gives

$$
\begin{aligned}
\operatorname{Var}\left[X_{t} \mid \mathcal{F}_{\leq s}\right] & =\frac{t-s}{1+\sigma s}\left(\sigma X_{s}^{2}+\eta X_{s}+1\right), \\
\operatorname{Var}\left[X_{t} \mid \mathcal{F}_{\geq u}\right] & =\frac{t(u-t)}{u+\tau}\left(\tau \frac{X_{u}^{2}}{u^{2}}+\theta \frac{X_{u}}{u}+1\right) .
\end{aligned}
$$

Theorem 2.5. If a square-integrable stochastic process $\left(X_{t}\right)$ with covariance (2.6) satisfies (2.7), (2.8), (2.27) and (2.28) with $\sigma, \tau \geq 0$ such that $\sigma \tau \leq 1 / 2^{4 r+10}$ for some $r>2$, then $\mathbb{E}\left(\left|X_{t}\right|^{r}\right)<\infty$ for all $t>0$. In particular, if $\sigma \tau=0$, then $\mathbb{E}\left(\left|X_{t}\right|^{r}\right)<\infty$ for all $r, t>0$.

\section{Proofs of the main Results}

3.1. Proof of Theorem 2.2, For $0<x<y<z$ define

$$
\begin{gathered}
\sigma_{y, x, z}:=\frac{A_{y, x, z}+B_{y, x, z}+C_{y, x, z}-1}{F_{y, x, z}}, \\
\tau_{y, x, z}:=\frac{x^{2} A_{y, x, z}+x z B_{y, x, z}+z^{2} C_{y, x, z}-y^{2}}{F_{y, x, z}}, \\
1+q_{y, x, z}:=\frac{B_{y, x, z}(z-x)}{F_{y, x, z}}, \eta_{y, x, z}:=\frac{D_{y, x, z}+E_{y, x, z}}{F_{y, x, z}}, \theta_{y, x, z}:=\frac{x D_{y, x, z}+z E_{y, x, z}}{F_{y, x, z}} .
\end{gathered}
$$

We will show that the left hand sides of the above equations do not depend on the arguments $y, x, z$. To this end we use three claims, proofs of which are given in the appendix.

Claim 3.1. For all $0<x<y<z$, and for $f=\sigma, \tau, q, \eta, \theta$ we have

$$
f_{y, x, z}=f_{\widetilde{y}, x, z},
$$

provided $x<\widetilde{y}<z$.

Claim 3.2. For all $0<x<y<z$, and $f=\sigma, \tau, q, \eta, \theta$ we have

$$
f_{y, x, z}=f_{y, \widetilde{x}, z}
$$

provided $0<\widetilde{x}<y$.

Claim 3.3. For all $0<x<y<z$, and $f=\sigma, \tau, q, \eta, \theta$ we have

$$
f_{y, x, z}=f_{y, x, \tilde{z}}
$$

provided $\widetilde{z}>y$.

It is easy to deduce that (3.1), (3.2) and (3.3) imply that functions $\sigma, \tau, q, \eta, \theta$ are in fact constants. Indeed, given $0<x_{1}<y_{1}<z_{1}$ and $0<x_{2}<y_{2}<z_{2}$, and 
$f=\sigma, \tau, q, \eta, \theta$, with $\widetilde{x}:=\min \left\{x_{1}, x_{2}\right\}$ and $\widetilde{z}:=\max \left\{z_{1}, z_{2}\right\}$, we see that

$$
\begin{aligned}
f_{y_{1}, x_{1}, z_{1}} & =f_{y_{1}, x_{1}, \tilde{z}} & & \text { by Claim } 3.3 \\
& =f_{y_{1}, \widetilde{x}, \tilde{z}} & & \text { by Claim } 3.2 \\
& =f_{y_{2}, \widetilde{x}, \tilde{z}} & & \text { by Claim } 3.1 \\
& =f_{y_{2}, x_{2}, \widetilde{z}} & & \text { by Claim } 3.2 \\
& =f_{y_{2}, x_{2}, z_{2}} & & \text { by Claim } 3.3
\end{aligned}
$$

(If $x_{2}=\widetilde{x}$ or $z_{2}=\widetilde{z}$, one or both of the last two steps is unnecessary.) So, for all $0<s<t<u$ we have

$$
\begin{gathered}
\sigma=\frac{A_{t, s, u}+B_{t, s, u}+C_{t, s, u}-1}{F_{t, s, u}}, \tau=\frac{s^{2} A_{t, s, u}+s u B_{t, s, u}+u^{2} C_{t, s, u}-t^{2}}{F_{t, s, u}}, \\
1+q=\frac{B_{t, s, u}(u-s)}{F_{t, s, u}}, \eta=\frac{D_{t, s, u}+E_{t, s, u}}{F_{t, s, u}}, \theta=\frac{s D_{t, s, u}+u E_{t, s, u}}{F_{t, s, u}} .
\end{gathered}
$$

The above equations, along with (2.13), form a system of linear equations in variables $A_{t, s, u}, \ldots, F_{t, s, u}$. This system must be solvable for all $0<s<t<u$, so its determinant $(u-s)^{3}(u(1+\sigma s)+\tau-q s) \neq 0$. As $u \rightarrow \infty, s \rightarrow 0$, the expression $u(1+\sigma s)+\tau-q s$ is positive. Thus $u(1+\sigma s)+\tau-q s>0$. Taking the limits $s \rightarrow 0, u \rightarrow 0, s=1, u \rightarrow \infty$, and $s \rightarrow u$, we get $\tau \geq 0, \sigma \geq 0$ and $q \leq 1+\sigma u+\tau / u$ respectively. Minimizing the latter over $u>0$ we get $q \leq 1+2 \sqrt{\sigma \tau}$. The unique solution of the system of equations is given by (2.15) and

$$
\begin{aligned}
A_{t, s, u} & =\frac{(u-t)[u(1+\sigma t)+\tau-q t]}{(u-s)[u(1+\sigma s)+\tau-q s]}, \\
B_{t, s, u} & =\frac{(u-t)(t-s)(1+q)}{(u-s)[u(1+\sigma s)+\tau-q s]}, \\
C_{t, s, u} & =\frac{(t-s)[t(1+\sigma s)+\tau-q s]}{(u-s)[u(1+\sigma s)+\tau-q s]}, \\
D_{t, s, u} & =\frac{(u-t)(t-s)(u \eta-\theta)}{(u-s)[u(1+\sigma s)+\tau-q s]}, \\
E_{t, s, u} & =\frac{(u-t)(t-s)(\theta-s \eta)}{(u-s)[u(1+\sigma s)+\tau-q s]} .
\end{aligned}
$$

A calculation shows that (2.14) holds.

\subsection{Proof of Theorem 2.3 ,}

Lemma 3.4. Under the assumptions of Theorem 2.3,

$$
\begin{array}{r}
\mathbf{C}_{t}=a_{t, s, u} \mathbf{C}_{s}+b_{t, s, u} \mathbf{C}_{u}, \\
\mathbf{C}_{t}^{2}=Q_{t, s, u}\left(\mathbf{C}_{s}, \mathbf{C}_{u}\right),
\end{array}
$$

where (compare (2.12))

$$
Q_{t, s, u}(\mathrm{x}, \mathrm{y})=A_{t, s, u} \mathrm{x}^{2}+B_{t, s, u} \mathrm{xy}+C_{t, s, u} \mathrm{y}^{2}+D_{t, s, u} \mathrm{x}+E_{t, s, u} \mathrm{y}+F_{t, s, u}
$$

with the coefficients given by (2.15) and (3.6)-(3.10). 
For the proof of Lemma 3.4, see the appendix. Note that from (3.11) it follows that $\mathbf{C}_{t}=t \mathrm{x}+\mathrm{y}$ for some infinite matrices $\mathrm{x}, \mathrm{y}$. Expanding the expressions such as $\mathbf{C}_{t}^{2}=t^{2} \mathrm{x}^{2}+t(\mathrm{xy}+\mathrm{yx})+\mathrm{y}^{2}$ on both sides of (3.12) we get

$$
\begin{aligned}
t^{2} \mathrm{x}^{2}+t(\mathrm{xy}+\mathrm{yx})+\mathrm{y}^{2} & =A_{t, s, u}\left(s^{2} \mathrm{x}^{2}+s(\mathrm{xy}+\mathrm{yx})+\mathrm{y}^{2}\right) \\
+B_{t, s, u}\left(\mathrm{y}^{2}+u \mathrm{yx}+\right. & \left.s \mathrm{x} \mathrm{y}+s u \mathrm{x}^{2}\right)+C_{t, s, u}\left(u^{2} \mathrm{x}^{2}+u(\mathrm{xy}+\mathrm{yx})+\mathrm{y}^{2}\right) \\
& +\left(D_{t, s, u}+E_{t, s, u}\right) \mathrm{y}+\left(s D_{t, s, u}+u E_{t, s, u}\right) \mathrm{x}+F_{t, s, u} .
\end{aligned}
$$

Applying the relations (2.13), (3.4), and (3.5) to the coefficients at each of the monomials in (3.14) we get

$$
\mathrm{xy}+\mathrm{yx}=\tau \mathrm{x}^{2}+\sigma \mathrm{y}^{2}+\eta \mathrm{y}+\theta \mathrm{x}+\mathrm{I}+(1+q) \mathrm{yx},
$$

which is the same as (1.1).

\subsection{Proof of Theorem 2.4 .}

3.3.1. Martingale property (2.16). We proceed by induction on $n$. Trivially, (2.16) holds for $n=0,1$; see (2.8). Assume then that $n>1$ and that (2.16) holds for $0,1, \ldots, n-1$ and all $0<s<t$.

\section{Lemma 3.5. Denoting}

$$
\mathcal{X}:=\mathbb{E}\left[p_{n}\left(X_{u} ; u\right) \mid \mathcal{F}_{\leq s}\right], \mathcal{Y}:=\mathbb{E}\left[p_{n}\left(X_{t} ; t\right) \mid \mathcal{F}_{\leq s}\right],
$$

we have

$$
b_{t, s, u} a_{n-1}(u) \mathcal{X}-a_{n-1}(t) \mathcal{Y}=-a_{t, s, u} a_{n-1}(s) p_{n}\left(X_{s} ; s\right)
$$

and

$$
\begin{aligned}
C_{t, s, u} a_{n-2}(u) a_{n-1}(u) \mathcal{X}-a_{n-2}(t) a_{n-1}(t) \mathcal{Y} \\
=-a_{n-1}(s) p_{n}\left(X_{s} ; s\right)\left(A_{t, s, u} a_{n-2}(s)+B_{t, s, u} a_{n-2}(u)\right)
\end{aligned}
$$

for all $n>1$.

The proof of this lemma is in the appendix. Subtracting equation (3.15) multiplied by $a_{n-2}(t)$ from (3.16), we get

$$
\begin{aligned}
& a_{n-1}(u)\left(C_{t, s, u} a_{n-2}(u)-b_{t, s, u} a_{n-2}(t)\right) \mathcal{X} \\
& \quad=-a_{n-1}(s) p_{n}\left(X_{s} ; s\right)\left(A_{t, s, u} a_{n-2}(s)+B_{t, s, u} a_{n-2}(u)-a_{t, s, u} a_{n-2}(t)\right) .
\end{aligned}
$$

Now we notice that

$$
\begin{aligned}
C_{t, s, u} a_{n-2}(u) & -b_{t, s, u} a_{n-2}(t) \\
= & -\frac{(t-s)(u-t)}{(u-s)[u(1+\sigma s)+\tau-q s]}\left[(q s-\tau) \sigma \alpha_{n-1}+(1+s \sigma) \beta_{n-1}\right] ;
\end{aligned}
$$

see (2.4), (2.20), and (3.8). Similarly,

$$
\begin{aligned}
A_{t, s, u} a_{n-2}(s) & +B_{t, s, u} a_{n-2}(u)-a_{t, s, u} a_{n-2}(t) \\
& =\frac{(t-s)(u-t)}{(u-s)[u(1+\sigma s)+\tau-q s]}\left[(q u-\tau) \sigma \alpha_{n-1}+(1+u \sigma) \beta_{n-1}\right] .
\end{aligned}
$$

Since (2.22) implies that

$$
\begin{aligned}
a_{n-1}(u)\left((q s-\tau) \sigma \alpha_{n-1}+\right. & \left.(1+s \sigma) \beta_{n-1}\right) \\
& =a_{n-1}(s)\left((q u-\tau) \sigma \alpha_{n-1}+(1+u \sigma) \beta_{n-1}\right)
\end{aligned}
$$


and $a_{n-1}(u) \neq 0$ by assumption, therefore, (3.17) becomes

$\left((q s-\tau) \sigma \alpha_{n-1}+(1+s \sigma) \beta_{n-1}\right) \mathcal{X}=\left((q s-\tau) \sigma \alpha_{n-1}+(1+s \sigma) \beta_{n-1}\right) p_{n}\left(X_{s} ; s\right)$.

It remains to verify that

$$
\left((q s-\tau) \sigma \alpha_{n-1}+(1+s \sigma) \beta_{n-1}\right) \neq 0 .
$$

Since $\beta_{n-1}=a_{n-1}(0) \neq 0$ by assumption, this is trivially true if $\sigma=0$. Suppose $\sigma>0$ and (3.20) is not true. Since $a_{n-1}(u) \neq 0$ from (3.18) we see that then the left hand side of (3.20) must vanish also when $s$ is replaced by $u$. Thus

$$
\sigma\left(\alpha_{n-1} q+\beta_{n-1}\right)=0, \beta_{n-1}-\sigma \tau \alpha_{n-1}=0 .
$$

Since $\beta_{n-1} \neq 0$, the determinant of the system of equations (3.21) is zero, $q+\sigma \tau=0$, and the second equation in (3.21) gives $\sigma \tau \neq 0$. Since $\alpha_{1}=0$, from (2.22) with $q=-\sigma \tau \neq 0$ we get $\alpha_{j}=\beta_{j}$ for all $j \geq 1$. As $\sigma \tau \neq 1$, this contradicts (3.21). Thus (3.20) holds. By (3.20), from (3.19) we get

$$
\mathcal{X}=p_{n}\left(X_{s} ; s\right),
$$

which means that $\left\{p_{n}(x ; t)\right\}_{t>0}$ is a martingale for every $n \geq 0$.

3.3.2. Orthogonality. Now we shall show that polynomials $\left\{p_{n}(x ; t)\right\}_{n}$ are orthogonal with respect to the distribution of $X_{t}$. Since the polynomials satisfy the three term recurrence (2.19), it suffices to show that for all $k=1,2, \ldots$,

$$
\mathbb{E}\left(p_{k}\left(X_{t} ; t\right)\right)=0 .
$$

Clearly, by the martingale property, $\mu_{k}=\mathbb{E}\left(p_{k}\left(X_{t} ; t\right)\right)$ does not depend on $t$. We proceed again by induction. From (4.2) we see that $a_{1}(t)=1+\sigma t$, so $(1+\sigma t) p_{2}(x ; t)=x^{2}-b_{1}(t) p_{1}(x ; t)-t$. Since $p_{1}(x ; t)=x$, assumption (2.6) implies that (3.22) holds for $k=1,2$. Assume then that for some $n \geq 3$ we have $\mu_{k}=0$ for all $1 \leq k \leq n-1$. By (2.19),

$$
\mathbb{E}\left(X_{t} p_{n}\left(X_{t} ; t\right)\right)=a_{n}(t) \mathbb{E}\left(p_{n+1}\left(X_{t} ; t\right)\right)=a_{n}(t) \mu_{n+1} .
$$

By (2.7), the martingale property of $\left\{p_{n}(x ; t)\right\}_{n}$, (2.19) and the induction assumption, the left hand side of the above equation transforms into

$$
\begin{aligned}
\mathbb{E}\left(X_{t} p_{n}\left(X_{t} ; t\right)\right) & =\mathbb{E}\left(\frac{t}{s} \mathbb{E}\left[X_{s} \mid \mathcal{F}_{\geq t}\right] p_{n}\left(X_{t} ; t\right)\right)=\frac{t}{s} \mathbb{E}\left(X_{s} \mathbb{E}\left[p_{n}\left(X_{t} ; t\right) \mid \mathcal{F}_{\leq s}\right]\right) \\
& =\frac{t}{s} \mathbb{E}\left(X_{s} p_{n}\left(X_{s} ; s\right)\right)=\frac{t}{s} a_{n}(s) \mathbb{E}\left(p_{n+1}\left(X_{s} ; s\right)\right)=\frac{t}{s} a_{n}(s) \mu_{n+1},
\end{aligned}
$$

so

$$
\left(\frac{a_{n}(s)}{s}-\frac{a_{n}(t)}{t}\right) \mu_{n+1}=0
$$

Since

$$
\frac{a_{n}(s)}{s}-\frac{a_{n}(t)}{t}=a_{n}(0) \frac{t-s}{t s} \neq 0,
$$

we get $\mu_{n+1}=\mathbb{E}\left(p_{n+1}\left(X_{t} ; t\right)\right)=0$, and the orthogonality of $\left\{p_{n}(x ; t)\right\}_{n}$ is proved. 
3.4. Proof of Theorem 2.5. The proof is based on the following two lemmas, the proofs of which are given in the appendix.

Lemma 3.6. Suppose $X, Y$ are square-integrable, standardized, and there are constants $\varepsilon, A, B \geq 0,0<\rho<1$ such that

$$
\begin{aligned}
& \mathbb{E}\left[(X-\rho Y)^{2} \mid Y\right] \leq A+B|Y|+\frac{\left(1-\rho^{2}\right) \varepsilon^{2}}{1+\varepsilon^{2}} Y^{2}, \\
& \mathbb{E}\left[(Y-\rho X)^{2} \mid X\right] \leq A+B|X|+\frac{\left(1-\rho^{2}\right) \varepsilon^{2}}{1+\varepsilon^{2}} X^{2} .
\end{aligned}
$$

If $2 \varepsilon<\rho<1$, then there are constants $C_{1}, C_{2}<\infty$ such that for $x>0$ we have

$$
\begin{aligned}
& \operatorname{Pr}(|X|>2 x / \rho)+\operatorname{Pr}(|Y|>2 x / \rho) \\
& \leq\left(C_{1} / x^{2}+C_{2} / x+\frac{4 \varepsilon^{2}}{\rho^{2}+\varepsilon^{2}} \frac{1+\rho}{1-\rho}\right)(\operatorname{Pr}(|X|>x)+\operatorname{Pr}(|Y|>x)) .
\end{aligned}
$$

Lemma 3.7. Suppose $X, Y$ are square-integrable, standardized, $\mathbb{E}\left(|X|^{p}\right)+\mathbb{E}\left(|Y|^{p}\right)<$ $\infty$ for some $p>1$ and the assumptions of Lemma 3.6 are satisfied with constants $\varepsilon>0,0<\rho<1$ such that

$$
2^{p+3} \varepsilon^{2} \frac{1+\rho}{1-\rho} \leq \rho^{p+3} .
$$

Then $\mathbb{E}\left(|X|^{p+1}\right)+\mathbb{E}\left(|Y|^{p+1}\right)<\infty$.

Proof of Theorem 2.5. Let $r>2$ be fixed. Since our proof relies only on inequalities, if $\sigma \tau=0$ we can increase slightly the values of the parameters $\sigma, \tau$ on the right hand sides of (2.28) and (2.27) to ensure $\sigma^{\prime}, \tau^{\prime}>0$ and $\sigma^{\prime} \tau^{\prime} \leq 2^{-4 r-10}$, replacing both equalities by the appropriate inequalities; thus without loss of generality we may assume that $\sigma \tau>0$.

Let $p=r-1>1$. Define $\varepsilon^{2}=\sqrt{\sigma \tau} / 2, t_{0}=\tau / \varepsilon^{2}$, and $s_{0}=\varepsilon^{2} / \sigma$. Then the correlation coefficient between $X_{s_{0}}$ and $X_{t_{0}}$ is $\rho=\sqrt{s_{0} / t_{0}}=\varepsilon^{2} / \sqrt{\sigma \tau}=1 / 2$. From the inequality form of (2.28) and (2.27) we deduce that random variables $X=X_{s_{0}} / \sqrt{s_{0}}$ and $Y=X_{t_{0}} / \sqrt{t_{0}}$ satisfy (3.23) and (3.24) with

$$
A=\frac{1-\rho^{2}}{1+\varepsilon^{2}}, \quad B=\frac{1-\rho^{2}}{1+\varepsilon^{2}} \max \{|\eta| \varepsilon / \sqrt{\sigma},|\theta| \sqrt{\tau} / \varepsilon\} .
$$

Condition (3.26) is satisfied since with $r=p+1$ we have $(\sigma \tau)^{1 / 2} \leq 1 / 2^{2 p+7}$. Thus

$$
2^{p+3} \varepsilon^{2} \frac{1+\rho}{1-\rho}=2^{p+3} 3 \varepsilon^{2}<2^{p+4}(\sigma \tau)^{1 / 2} \leq 1 / 2^{p+3}=\rho^{p+3} .
$$

Therefore, by Lemma 3.7 $\mathbb{E}\left(\left|X_{t_{0}}\right|^{r}\right)=\mathbb{E}\left(\left|X_{t_{0}}\right|^{p+1}\right)<\infty$. Observe that if $\mathbb{E}\left(\left|X_{t_{0}}\right|^{r}\right)$ $<\infty$ for some $t_{0}>0, r>1$, then $\mathbb{E}\left(\left|X_{t}\right|^{r}\right)<\infty$ for all $t>0$. Indeed, if $\mathbb{E}\left(\left|X_{t_{0}}\right|^{r}\right)<$ $\infty$ then $\mathbb{E}\left(\left|X_{s}\right|^{r}\right)<\infty$ for all $s<t$ by (2.8). Similarly, $\mathbb{E}\left(\left|X_{u}\right|^{r}\right)<\infty$ for all $u>t$ by (2.7). Therefore, $\mathbb{E}\left(\left|X_{t}\right|^{r}\right)<\infty$ for all $t \geq 0$.

\section{EXAMPLES OF EXPLICIT RECURRENCES}

In this section we will call $\left(X_{t}\right)$ a quadratic harness with parameters $q, \eta, \theta, \sigma, \tau$ if (2.3), (2.6), and (2.14) hold. Our goal is to derive explicit versions of (2.19) at the expense of additional assumptions on the parameters. 
Theorem 4.1. Suppose $\left(X_{t}\right)$ is a quadratic harness with parameters $0 \leq \sigma \tau<1$, $-1<q \leq 1-2 \sqrt{\sigma \tau}$. Moreover, assume that for each $t>0$, the random variable $X_{t}$ has moments of all orders and infinite support. Then $\left(X_{t}\right)$ has orthogonal martingale polynomials which are given by recurrence (2.19) with $p_{0}(x, t)=1, p_{1}(x, t)=x$, where the coefficients of the recurrence are linear functions of $t$,

$$
a_{n}(t)=\sigma \alpha_{n+1} t+\beta_{n+1}, \quad b_{n}(t)=\gamma_{n} t+\delta_{n}, \quad c_{n}(t)=\left(\beta_{n} t+\tau \alpha_{n}\right) \omega_{n},
$$

which are determined as follows.

(1) The initial conditions are

$$
\alpha_{1}=0, \beta_{1}=1, \gamma_{0}=\delta_{0}=0, \omega_{1}=1 .
$$

(2) Sequences $\left(\alpha_{n}\right),\left(\beta_{n}\right)$ satisfy

$$
\left[\begin{array}{c}
\alpha_{n+1} \\
\beta_{n+1}
\end{array}\right]=\left[\begin{array}{cc}
q & 1 \\
-\sigma \tau & 1
\end{array}\right] \times\left[\begin{array}{l}
\alpha_{n} \\
\beta_{n}
\end{array}\right], \quad n \geq 1
$$

Moreover, denoting

$$
\lambda_{n, k}=\beta_{n} \beta_{n+k}-\sigma \tau \alpha_{n} \alpha_{n+k}
$$

we have $\lambda_{n, k}>0$ for all $n \geq 1, k \geq 0$.

(3) Setting $\lambda_{0,2}=\alpha_{0}=\beta_{0}=0$, sequences $\left(\gamma_{n}\right)$, ( $\left.\delta_{n}\right)$ satisfy the following system of linear recurrences:

$$
\begin{aligned}
\gamma_{n+1}= & \frac{q+\sigma \tau}{\lambda_{n+2,0}}\left(\lambda_{n, 2} \gamma_{n}+\left(\alpha_{n+2} \beta_{n}-\beta_{n+2} \alpha_{n}\right) \sigma \delta_{n}\right) \\
& +\frac{\sigma \alpha_{n+2}}{\lambda_{n+2,0}}\left(\eta \tau \alpha_{n+1}+\theta \beta_{n+1}\right)+\frac{\beta_{n+2}}{\lambda_{n+2,0}}\left(\theta \sigma \alpha_{n+1}+\eta \beta_{n+1}\right), \\
\delta_{n+1}= & \frac{q+\sigma \tau}{\lambda_{n+2,0}}\left(\lambda_{n, 2} \delta_{n}+\left(\alpha_{n+2} \beta_{n}-\beta_{n+2} \alpha_{n}\right) \tau \gamma_{n}\right) \\
& +\frac{\beta_{n+2}}{\lambda_{n+2,0}}\left(\eta \tau \alpha_{n+1}+\theta \beta_{n+1}\right)+\frac{\tau \alpha_{n+2}}{\lambda_{n+2,0}}\left(\theta \sigma \alpha_{n+1}+\eta \beta_{n+1}\right), n \geq 0 .
\end{aligned}
$$

(4) Sequence $\left(\omega_{n}\right)$ satisfies the linear recurrence

$$
\begin{aligned}
\omega_{n+1}= & \frac{(q+\sigma \tau) \lambda_{n-1,1}}{\lambda_{n+1,1}} \omega_{n} \\
& +\frac{1+\gamma_{n}\left(\tau \gamma_{n}-\delta_{n}+\theta\right)+\delta_{n}\left(q \gamma_{n}+\sigma \delta_{n}+\eta\right)}{\lambda_{n+1,1}}, n \geq 2
\end{aligned}
$$

with the initial term

$$
\omega_{2}=(1+q) \frac{(1-\sigma \tau)^{2}+(\eta+\theta \sigma)(\theta+\eta \tau)}{(1-\sigma \tau)^{2}(1-\sigma \tau(2+q))} .
$$

Remark 4.1. If $-1 \leq q<1-2 \sqrt{\sigma \tau}$, then the explicit solution of recurrence (4.2) is

$$
\alpha_{n}=\frac{\lambda_{+}^{n-1}-\lambda_{-}^{n-1}}{\lambda_{+}-\lambda_{-}}, \beta_{n}=\frac{\lambda_{+}^{n-1}\left(1-\lambda_{-}\right)+\lambda_{-}^{n-1}\left(\lambda_{+}-1\right)}{\lambda_{+}-\lambda_{-}},
$$

where

$$
\lambda_{ \pm}=\frac{1}{2}\left(1+q \pm \sqrt{(1+q)^{2}-4(q+\sigma \tau)}\right) .
$$

The proof of Theorem 4.1 relies on Theorem 2.4 and the following lemma. 
Lemma 4.2. If $\sigma \tau<1$ and $-1 \leq q \leq 1-2 \sqrt{\sigma \tau}$, then the solutions of (4.2) satisfy

$$
\beta_{n}>\sqrt{\sigma \tau} \alpha_{n} \geq 0 \text {. }
$$

In particular,

$$
\beta_{n} \beta_{n+1}>\sigma \tau \alpha_{n} \alpha_{n+1}, \beta_{n}^{2}>\sigma \tau \alpha_{n}^{2}, \text { for all } n \geq 1 .
$$

Proof. From the explicit solution in Remark 4.1, we see that $\left|\lambda_{-}\right| \leq\left|\lambda_{+}\right|$and hence $\alpha_{n} \geq 0$ for $n>1$. We now prove by induction that $\beta_{n}>\sqrt{\sigma \tau} \alpha_{n}$ for all $n$. This is trivially true for $n=1$; see (2.21). Suppose the inequality is satisfied for some $n \geq 1$. Then (4.2) implies that $\beta_{n+1}-\sqrt{\sigma \tau} \alpha_{n+1}=(1-\sqrt{\sigma \tau}) \beta_{n}-\sqrt{\sigma \tau}(q+\sqrt{\sigma \tau}) \alpha_{n} \geq$ $(1-\sqrt{\sigma \tau})\left(\beta_{n}-\sqrt{\sigma \tau} \alpha_{n}\right)>0$.

Proof of Theorem 4.1. By Lemma 4.2, $a_{n}(t)=\sigma \alpha_{n+1} t+\beta_{n+1} \geq \beta_{n+1}>0$ for all $t \geq 0, n \geq 0$. Moreover, recurrences (4.3), (4.4) are well defined and have a unique solution. Therefore, to end the proof we need only to verify that with

$$
\varepsilon_{n}=\omega_{n} \beta_{n}, \varphi_{n}=\tau \omega_{n} \alpha_{n},
$$

the equations (2.21)-(2.26) are satisfied. Trivially, (2.21) holds true. It is easy to verify that the solution of (4.2) satisfies (2.22). Using (4.6) from (4.2) we also get (2.26). It remains to show that equations (2.23), (2.24), and (2.25) hold. We will rewrite these equations using (4.2) and its equivalent form

$$
q \beta_{n+1}+\sigma \tau \alpha_{n+1}=(q+\sigma \tau) \beta_{n}, \beta_{n+1}-\alpha_{n+1}=-(q+\sigma \tau) \alpha_{n}, n \geq 1 .
$$

Equation (2.23) is equivalent to

$$
\begin{aligned}
& \gamma_{n+1}\left(\beta_{n+1}-\sigma \tau \alpha_{n+1}\right)-\delta_{n+1} \sigma\left(\beta_{n+1}+\alpha_{n+1} q\right) \\
& =\left(\sigma \tau \alpha_{n+1}+\beta_{n+1} q\right) \gamma_{n}+\delta_{n} \sigma\left(\beta_{n+1}-\alpha_{n+1}\right)+\sigma \alpha_{n+1} \theta+\beta_{n+1} \eta .
\end{aligned}
$$

Using (4.2) and (4.7), we can rewrite it as

$$
\gamma_{n+1} \beta_{n+2}-\sigma \delta_{n+1} \alpha_{n+2}=(q+\sigma \tau) \beta_{n} \gamma_{n}-(q+\sigma \tau) \sigma \alpha_{n} \delta_{n}+\sigma \alpha_{n+1} \theta+\beta_{n+1} \eta
$$

Similarly, (2.25) for our sequences can be rewritten as

$$
\begin{aligned}
& \omega_{n}\left(\delta_{n+1}\left(\beta_{n+1}-\sigma \tau \alpha_{n+1}\right)-\tau \gamma_{n+1}\left(\beta_{n+1}+q \alpha_{n+1}\right)\right) \\
& \quad=\omega_{n}\left(\left(q \beta_{n+1}+\sigma \tau \alpha_{n+1}\right) \delta_{n}+\tau\left(\beta_{n+1}-\alpha_{n+1}\right) \gamma_{n}+\theta \beta_{n+1}+\eta \tau \alpha_{n+1}\right) .
\end{aligned}
$$

Equations (4.8), (4.9) are satisfied; in fact, (4.3) was obtained by solving this system of equations when $\omega_{n}>0$. Thus (2.23) and (2.25) hold. Substituting (4.6) into (2.24) and using (4.2) and Lemma 4.2 we get

$$
\begin{gathered}
\omega_{n+1}=\frac{\beta_{n}\left(q \beta_{n}+\sigma \tau \alpha_{n}\right)+\sigma \tau \alpha_{n}\left(\beta_{n}-\alpha_{n}\right)}{\beta_{n+1} \beta_{n+2}-\sigma \tau \alpha_{n+1} \alpha_{n+2}} \omega_{n} \\
\quad+\frac{1+\gamma_{n}\left(\tau \gamma_{n}-\delta_{n}+\theta\right)+\delta_{n}\left(q \gamma_{n}+\sigma \delta_{n}+\eta\right)}{\beta_{n+1} \beta_{n+2}-\sigma \tau \alpha_{n+1} \alpha_{n+2}} .
\end{gathered}
$$

Since (4.7) holds, thus (2.24) is equivalent to (4.4).

Remark 4.2. From (4.3) we get

$$
\gamma_{1}=\frac{\eta+\theta \sigma}{1-\sigma \tau}, \quad \delta_{1}=\frac{\eta \tau+\theta}{1-\sigma \tau}
$$


which implies that the initial recurrences are

$$
\begin{aligned}
& x p_{0}(x ; t)=p_{1}(x ; t), \\
& x p_{1}(x ; t)=(1+\sigma t) p_{2}(x ; t)+\frac{(\eta+\theta \sigma) t+(\eta \tau+\theta)}{1-\sigma \tau} p_{1}(x ; t)+t p_{0}(x ; t) .
\end{aligned}
$$

Thus the first three orthogonal martingale polynomials are

$$
\begin{aligned}
& p_{0}(x ; t)=1, p_{1}(x ; t)=x, \\
& p_{2}(x ; t)=\frac{1}{1+\sigma t} x^{2}-\frac{(\eta+\theta \sigma) t+\eta \tau+\theta}{(1-\sigma \tau)(1+\sigma t)} x-\frac{t}{1+\sigma t} .
\end{aligned}
$$

4.1. Free quadratic harnesses. Free harnesses have parameter

$$
q=-\sigma \tau .
$$

The adjective "free" is motivated by the fact that when $\sigma \tau=0$ this choice is related to free convolutions that arise in free probability; see [14, Section 4.3] and 12, Section 4]. In general, from Theorem 4.1 it is clear that this choice of $q$ significantly simplifies the recurrences. It is not obvious whether this case is further related to free convolutions; see however [26].

Assuming $\sigma \tau<1$, (4.14) implies that $-1<q<1-2 \sqrt{\sigma \tau}$, so it follows from Theorem 4.1 that the orthogonal martingale polynomials exist. It is easy to check that the solution of (4.2) is

$$
\alpha_{n}=(1-\sigma \tau)^{n-2}, \beta_{n}=(1-\sigma \tau)^{n-2}, n \geq 2 .
$$

For our choice of $q$ and $n \geq 0$ we have

$$
\lambda_{n+2,0}=\beta_{n+2}^{2}-\sigma \tau \alpha_{n+2}^{2}=(1-\sigma \tau)^{2 n+1} .
$$

Using this identity, from (4.3) we get for $n \geq 2$,

$$
\begin{gathered}
\gamma_{n}=\frac{\eta+2 \theta \sigma+\eta \sigma \tau}{(1-\sigma \tau)^{2}}, \\
\delta_{n}=\frac{\theta+2 \eta \tau+\theta \sigma \tau}{(1-\sigma \tau)^{2}} .
\end{gathered}
$$

Similarly,

$$
\lambda_{n+1,1}=\beta_{n+1} \beta_{n+2}-\sigma \tau \alpha_{n+1} \alpha_{n+2}=(1-\sigma \tau)^{2 n}, n \geq 0 .
$$

Substituting this and $\delta_{n}, \gamma_{n}$ into (4.4), we get

$$
\omega_{n}=\frac{(1-\sigma \tau)^{2}+(\eta+\theta \sigma)(\theta+\eta \tau)}{(1-\sigma \tau)^{2 n}}, n \geq 3
$$

We also compute

$$
\omega_{2}=\frac{(1-\sigma \tau)^{2}+(\eta+\theta \sigma)(\theta+\eta \tau)}{(1-\sigma \tau)^{3}} .
$$


Therefore recurrence (2.19) with initial values (2.21) gives

$$
\begin{gathered}
x p_{2}(x)=(1-\sigma \tau)(1+\sigma t) p_{3}(x)+\frac{(\eta+2 \theta \sigma+\eta \sigma \tau) t+\theta+2 \eta \tau+\theta \sigma \tau}{(1-\sigma \tau)^{2}} p_{2}(x) \\
+\frac{(1-\sigma \tau)^{2}+(\eta+\theta \sigma)(\theta+\eta \tau)}{(1-\sigma \tau)^{3}}(t+\tau) p_{1}(x) \\
x p_{n}(x)=(1-\sigma \tau)^{n-1}(1+\sigma t) p_{n+1}(x)+\frac{(\eta+2 \theta \sigma+\eta \sigma \tau) t+\theta+2 \eta \tau+\theta \sigma \tau}{(1-\sigma \tau)^{2}} p_{n}(x) \\
+\frac{(1-\sigma \tau)^{2}+(\eta+\theta \sigma)(\theta+\eta \tau)}{(1-\sigma \tau)^{n+2}}(t+\tau) p_{n-1}(x)
\end{gathered}
$$

for $n \geq 2$. After renormalizing the $n$-th polynomial by $(1-\sigma \tau)^{(n-2)(n-1) / 2}$, and denoting

$$
\gamma=\gamma_{1}, \delta=\delta_{1}
$$

see (4.10), from Theorem 4.1 we get the following.

Proposition 4.3 (Free quadratic harnesses). Suppose $\left(X_{t}\right)$ is a quadratic harness with parameters such that $1+\gamma \delta>0,0 \leq \sigma \tau<1$, and $q=-\sigma \tau$. If for $t>0$, the random variable $X_{t}$ has all moments and infinite support, then it has orthogonal martingale polynomials given by the three step recurrences

$$
\begin{aligned}
x p_{0}(x ; t)= & p_{1}(x ; t) \\
x p_{1}(x ; t)= & (1+\sigma t) p_{2}+(\gamma t+\delta) p_{1}(x ; t)+t p_{0}(x ; t) \\
x p_{2}(x ; t)= & (1+\sigma t) p_{3}(x ; t)+\frac{(\gamma+\sigma \delta) t+\delta+\tau \gamma}{1-\sigma \tau} p_{2}(x ; t)+\frac{1+\gamma \delta}{1-\sigma \tau}(t+\tau) p_{1}(x ; t) \\
x p_{n}(x ; t)= & (1+\sigma t) p_{n+1}(x ; t)+\frac{(\gamma+\sigma \delta) t+\delta+\tau \gamma}{1-\sigma \tau} p_{n}(x ; t) \\
& +\frac{1+\gamma \delta}{(1-\sigma \tau)^{2}}(t+\tau) p_{n-1}(x ; t), n \geq 3 .
\end{aligned}
$$

Remark 4.3. The recurrence in Proposition 4.3 is a finite perturbation of the constant coefficient recurrence which was analyzed by many authors; see [34 and the references therein, and see also [32] and [5, (4.29)].

Remark 4.4. In [12] we show that the free bi-Poisson process is associated with the generalized free convolution studied in [8]. It is interesting to ask if an analogous situation occurs for the general free harnesses of Proposition 4.3. for recent extensions of generalized free convolutions to perturbations of higher-order terms, see 26].

4.2. Classical quadratic harnesses. The classical quadratic harnesses have parameter $q=1-2 \sqrt{\sigma \tau}$. The adjective "classical" is motivated by the fact that when $\sigma \tau=0$, quadratic harnesses with $q=1$ are related to classical stochastic processes; see [14, Section 4.2].

Proposition 4.4 (Classical quadratic harnesses). Suppose $\left(X_{t}\right)$ is a quadratic harness with parameters such that $(1-\sigma \tau)^{2}+(\eta+\theta \sigma)(\theta+\eta \tau)>0,0 \leq \sigma \tau<1$, and $q=1-2 \sqrt{\sigma \tau}$. If for $t>0$, the random variable $X_{t}$ has all moments and infinite support, then it has orthogonal martingale polynomials given by the three step 
recurrences (4.11), (4.12), and

$$
\begin{aligned}
x p_{n}(x ; t)= & (1+(n-1) \rho+n \sigma t) p_{n+1}(x ; t)+\left(\gamma_{n} t+\delta_{n}\right) p_{n}(x ; t) \\
& +\omega_{n}^{\circ}((1+(n-2) \rho) t+\tau(n-1)) p_{n-1}(x ; t), n \geq 2,
\end{aligned}
$$

where $\rho=\sqrt{\sigma \tau}$,

$$
\begin{aligned}
\gamma_{n}= & \frac{n(1+(n-2) \rho)}{(1-\rho)^{2}(1+(2 n-1) \rho)(1+(2 n-3) \rho)} \times(\eta+(2 n-1) \theta \sigma \\
& \left.+(2 n-3) \eta \rho+2(n-1)^{2} \eta \rho^{2}+\left(2(n-1)^{2}-1\right) \theta \sigma \rho\right), n \geq 1,
\end{aligned}
$$

$$
\begin{aligned}
& \delta_{n}=\frac{n(1+(n-2) \rho)}{(1-\rho)^{2}(1+(2 n-1) \rho)(1+(2 n-3) \rho)} \times(\theta+(2 n-1) \eta \tau \\
&\left.+(2 n-3) \theta \rho+2(n-1)^{2} \theta \rho^{2}+\left(2(n-1)^{2}-1\right) \eta \tau \rho\right), n \geq 1,
\end{aligned}
$$

$$
\begin{aligned}
\omega_{n}^{\circ}= & \frac{n(1+(n-3) \rho)}{(1-\rho)^{2}(1+(2 n-2) \rho)(1+(2 n-4) \rho)} \\
& +\frac{n(n-1)(1+(n-2) \rho)(1+(n-3) \rho)}{(1-\rho)^{4}(1+(2 n-2) \rho)(1+(2 n-3) \rho)^{2}(1+(2 n-4) \rho)} \\
& \quad \times((1+(n-2) \rho) \theta+(n-1) \eta \tau)((1+(n-2) \rho) \eta+(n-1) \theta \sigma), n \geq 2 .
\end{aligned}
$$

Proof. The assumptions of Theorem 4.1 are satisfied, so the orthogonal martingale polynomials exist, and we only need to solve the recurrences (4.2), (4.3), (4.4), and renormalize the polynomials to simplify the final three step recurrence (4.16). From (4.2) we get

$$
\begin{gathered}
\alpha_{n}=(1-\rho)^{n-2}(n-1), n \geq 1, \\
\beta_{n}=(1-\rho)^{n-2}(1+(n-2) \rho), n \geq 2 .
\end{gathered}
$$

Thus $\lambda_{1, k}=\beta_{k+1}, k \geq 0$, and a calculation gives

$$
\begin{gathered}
\lambda_{n, k}=(1-\rho)^{2 n+k-3}(1+(2 n+k-3) \rho), n \geq 2, \\
\alpha_{n+2} \beta_{n}-\beta_{n+2} \alpha_{n}=2(1-\rho)^{2 n-1}, n \geq 1 .
\end{gathered}
$$

Since $q+\sigma \tau=(1-\rho)^{2}$, equations (4.3) simplify to

$$
\begin{aligned}
\gamma_{n+1}= & \frac{1}{1+(2 n+1) \rho} \gamma_{n}+\frac{2 \sigma}{1+(2 n+1) \rho} \delta_{n} \\
& +\frac{\left(1+(2 n-1) \rho+2 n^{2} \rho^{2}\right) \eta+\left(2 n+1+\left(2 n^{2}-1\right) \rho\right) \theta \sigma}{(1-\rho)^{2}(1+(2 n+1) \rho)}, \\
\delta_{n+1}= & \frac{1+(2 n-1) \rho}{1+(2 n+1) \rho} \delta_{n}+\frac{2 \tau}{1+(2 n+1) \rho} \gamma_{n} \\
& +\frac{\left(1+(2 n-1) \rho+2 n^{2} \rho^{2}\right) \theta+\left(2 n+1+\left(2 n^{2}-1\right) \rho\right) \eta \tau}{(1-\rho)^{2}(1+(2 n+1) \rho)} .
\end{aligned}
$$

Formula (4.10) shows that (4.17) and (4.18) hold true for $n=1$. Assuming that (4.17) and (4.18) hold true for some $n \geq 1$, from equations (4.21) and (4.22) a 
computer-assisted calculation shows that the formulas hold true for $n+1$ as well. We now show that the solution of (4.4) is

$$
\begin{aligned}
\omega_{n}= & \frac{n(1+(n-3) \rho)}{(1-\rho)^{2 n-2}(1+(2 n-2) \rho)(1+(2 n-4) \rho)} \\
& +\frac{n(n-1)(1+(n-2) \rho)(1+(n-3) \rho)}{(1-\rho)^{2 n}(1+(2 n-2) \rho)(1+(2 n-3) \rho)^{2}(1+(2 n-4) \rho)} \\
& \times((1+(n-2) \rho) \theta+(n-1) \eta \tau)((1+(n-2) \rho) \eta+(n-1) \theta \sigma), n \geq 1 .
\end{aligned}
$$

Indeed, a calculation shows that the formula holds true for $n=1,2$. Suppose (4.23) holds for some $n \geq 2$. For $k=1$, formula (4.20) holds also for $n=1$; thus for $n \geq 2$, recurrence (4.4) simplifies to

$$
\omega_{n+1}=\frac{1+(2 n-4) \rho}{(1-\rho)^{2}(1+2 n \rho)} \omega_{n}+\frac{1+\tau \gamma_{n}^{2}+\sigma \delta_{n}^{2}+\theta \gamma_{n}+\eta \delta_{n}-2 \rho \delta_{n} \gamma_{n}}{(1-\rho)^{2 n}(1+2 n \rho)} .
$$

Using (4.21), (4.22) and (4.23), a computer assisted calculation verifies that (4.23) holds true for $n+1$. Renormalizing the $n$-th polynomial in (2.19) by the factor $(1-\rho)^{(n-2)(n-1) / 2}, n \geq 1$, we get (4.16) with $\omega_{n}$ replaced by $\omega_{n}^{\circ}$.

4.3. Orthogonal martingale polynomials when $\sigma \tau=0$. We use the standard $q$-notation

$$
\begin{aligned}
{[n]_{q} } & =1+q+\cdots+q^{n-1}, \\
{[n]_{q} ! } & =[1]_{q}[2]_{q} \cdots[n]_{q},
\end{aligned}
$$

with the usual conventions $[0]_{q}=0,[0]_{q} !=1$. In this notation, Remark 4.1 gives $\alpha_{n}=[n-1]_{q}$, and $\beta_{n}=1$. Passing to the time-inverse $\left(t X_{1 / t}\right)$ if necessary, without loss of generality we may assume that $\sigma=0$. In this case, recurrences in Theorem 4.1 have explicit solutions and orthogonal martingale polynomials are monic.

Theorem 4.5. Suppose $\left(X_{t}\right)$ is a quadratic harness with covariance (2.6) and parameters such that $\sigma=0,-1<q \leq 1$, and $1+[n]_{q} \eta \theta+[n]_{q}^{2} \tau \eta^{2}>0$ for all $n$. If for each $t>0$ the random variable $X_{t}$ has infinite support, then the monic orthogonal martingale polynomials $p_{n}(x ; t)$ are given by the recurrence

$$
\begin{aligned}
& x p_{n}(x ; t)=p_{n+1}(x ; t)+\left(\eta t+\theta+\left([n]_{q}+[n-1]_{q}\right) \eta \tau\right)[n]_{q} p_{n}(x ; t) \\
& \quad+\left(t+\tau[n-1]_{q}\right)\left(1+[n-1]_{q} \eta \theta+[n-1]_{q}^{2} \tau \eta^{2}\right)[n]_{q} p_{n-1}(x ; t), n \geq 1,
\end{aligned}
$$

with the initial condition $p_{0}=1, p_{1}(x)=x$.

Remark 4.5. It is easy to give simple sufficient conditions on the parameters which imply that $\omega_{n}:=1+[n-1]_{q} \eta \theta+[n-1]_{q}^{2} \tau \eta^{2}>0$ for all $n \geq 1$. Suppose that $-1<q \leq 1$.

(1) If $\eta \theta \geq 0$, then, trivially, $\omega_{n}>0$.

(2) If $\tau=0$, then $\omega_{n}>0$ provided $1+\eta \theta>\max \{q, 0\}$. Indeed, for $n \geq 2$ we have

$$
\begin{aligned}
1+q \leq[n]_{q} \leq 1 & \text { if }-1<q<0, \\
1 \leq[n]_{q}<1 /(1-q) & \text { if } 0 \leq q<1 .
\end{aligned}
$$

For $\eta \theta<0$ we get $\omega_{n}>0$ from the right hand sides of these equations. 
(3) If $-1<q<1$ and $\theta^{2}<4 \tau$, then $\omega_{n}>0$. Indeed, then the quadratic function $f(x)=1+x \eta \theta+x^{2} \tau \eta^{2}$ is non-negative.

(4) If $\tau>0, \eta \theta<0,1+\eta \theta+\tau \eta^{2}>0$, and $\theta^{2} \geq 4 \tau$, then the sufficient condition is

$$
1+\min \{q, 0\}>\frac{|\theta|+\sqrt{\theta^{2}-4 \tau}}{2 \tau|\eta|} .
$$

Indeed, inequality $1+\eta \theta+\tau \eta^{2}>0$ implies $\omega_{2}>0$. Considering separately the cases $\theta<0$ and $\theta>0$, the larger root of $1+x \eta \theta+x^{2} \tau \eta^{2}=0$ is $\frac{|\theta|+\sqrt{\theta^{2}-4 \tau}}{2 \tau|\eta|}$. If (4.27) holds, then the left hand sides of inequalities (4.25), (4.26) imply $\omega_{n}>0$ for $n \geq 3$.

It might be interesting to point out the explicit recurrence for the case $\tau=0$.

Corollary 4.6. Suppose $\left(X_{t}\right)$ is a quadratic harness with covariance (2.6) and parameters such that $\tau=0,-1<q<1$, and $1+[n]_{q} \eta \theta+[n]_{q}^{2} \sigma \theta^{2}>0$ for all $n$. Suppose that for $t>0$ the random variable $X_{t}$ has infinite support. Then as the orthogonal martingale polynomials for $\left(X_{t}\right)$ we can take polynomials $p_{n}(x ; t)$ given by the recurrence

$$
\begin{gathered}
x p_{n}(x ; t)=\left(1+t \sigma[n]_{q}\right) p_{n+1}(x ; t)+\left(\theta+t \eta+\left([n]_{q}+[n-1]_{q}\right) \theta \sigma t\right)[n]_{q} p_{n}(x ; t) \\
+t\left(1+[n-1]_{q} \eta \theta+[n-1]_{q}^{2} \sigma \theta^{2}\right)[n]_{q} p_{n-1}(x ; t)
\end{gathered}
$$

Proof of Theorem 4.5. We use the notation from (2.20). The integrability assumption of Theorem 4.1 is fulfilled by Theorem 2.5 and the system of recurrences in Theorem 4.1 simplifies. Since $\alpha_{n}=[n-1]_{q}, \beta_{n}=1$, the first equation of recurrence (4.3) becomes

$$
\gamma_{n+1}=\eta+\gamma_{n} q
$$

which gives

$$
\gamma_{n}=\eta[n]_{q}
$$

The other recurrences are solved inductively. Suppose that

$$
\begin{aligned}
\delta_{n-1} & =\left(\theta+\eta \tau\left([n-1]_{q}+[n-2]_{q}\right)\right)[n-1]_{q} \\
\omega_{n} & =\left(1+[n-1]_{q} \eta \theta+[n-1]_{q}^{2} \tau \eta^{2}\right)[n]_{q}
\end{aligned}
$$

hold. The initial conditions say that both formulas are satisfied for $n=1$, and the second one holds true also for $n=2$. We use (4.28) and (4.29) to compute $\delta_{n}$ from the second equation in (4.3) as follows:

$$
\begin{gathered}
\delta_{n}=\theta+q \delta_{n-1}+q\left([n]_{q}-[n-2]_{q}\right) \tau \gamma_{n-1}+\eta \tau[n-1]_{q}+\eta \tau[n]_{q}=\theta\left(1+q[n-1]_{q}\right) \\
+\eta \tau\left(q\left([n-1]_{q}+[n-2]_{q}\right)[n-1]_{q}+q\left([n]_{q}-[n-2]_{q}\right)[n-1]_{q}+[n-1]_{q}+[n]_{q}\right) \\
=\theta[n]_{q}+\eta \tau\left(q\left([n-1]_{q}+[n]_{q}\right)[n-1]_{q}+[n-1]_{q}+[n]_{q}\right) \\
=\theta[n]_{q}+\eta \tau\left([n-1]_{q}+[n]_{q}\right)\left(1+q[n-1]_{q}\right)=\theta[n]_{q}+\eta \tau\left([n-1]_{q}+[n]_{q}\right)[n]_{q} .
\end{gathered}
$$

Finally, (4.4) determines $\omega_{n+1}$ as

$$
\omega_{n+1}=q \omega_{n}+\gamma_{n}^{2} \tau+q \gamma_{n} \delta_{n}+\gamma_{n} \theta+\delta_{n} \eta+1-\gamma_{n} \delta_{n}
$$


Using the inductive assumption (4.30), and already established formulas (4.28) and (4.29), we get

$$
\begin{gathered}
\omega_{n+1}=\left(1+q[n]_{q}\right)+\left(1+q[n-1]_{q}\right)[n]_{q} \eta \theta+q[n-1]_{q}^{2}[n]_{q} \tau \eta^{2}+\tau \eta^{2}[n]_{q}^{2} \\
+\eta\left(1+q[n]_{q}\right) \delta_{n}-\eta[n]_{q} \delta_{n}=[n+1]_{q}+[n]_{q}^{2} \eta \theta+q[n-1]_{q}^{2}[n]_{q} \tau \eta^{2}+\tau \eta^{2}[n]_{q}^{2}+\eta q^{n} \delta_{n} \\
=[n+1]_{q}+[n+1]_{q}[n]_{q} \eta \theta+\tau \eta^{2}\left(q[n-1]_{q}[n]_{q}\left([n-1]_{q}+q^{n-1}\right)+[n]_{q}^{2}+q^{n}[n]_{q}^{2}\right) .
\end{gathered}
$$

The coefficient at $\tau \eta^{2}$ simplifies to

$$
q[n-1]_{q}[n]_{q}^{2}+[n]_{q}^{2}+q^{n}[n]_{q}^{2}=[n+1]_{q}[n]_{q}^{2} .
$$

Thus $\omega_{n+1}=\left(1+[n]_{q} \eta \theta+[n]_{q}^{2} \tau \eta^{2}\right)[n+1]_{q}$. Formula (4.24) comes from (2.19) after substituting (4.28)-(4.30) into (2.20).

4.4. Operator solutions. In this section we re-derive the recurrences for some special orthogonal martingale polynomials from Section 4.3 by an operator approach which is related to Lie algebra techniques. This method has a more ad hoc character, so we concentrate on two relatively simple cases only.

In the operator approach, we go back directly to Theorem 2.3. We re-interpret the matrices $x, y$ as the linear operators acting on the formal power series in an auxiliary variable $z$. This identifies the martingale polynomial $p_{n}(x ; t)$ with $z^{n}$, where $z$ is an auxiliary variable; a similar technique appeared in umbral calculus 33, Ch. 1], in orthogonal polynomials 24, and in the Segal-Bargmann representation 31. We seek the solutions of (1.1) in terms of the $q$-differentiation operator

$$
\mathrm{D}_{q}(g)(z)= \begin{cases}\frac{g(z)-g(q z)}{(1-q) z} & q \neq 1, \\ g^{\prime}(z) & q=1\end{cases}
$$

and the multiplication operator

$$
\mathrm{Z}(g)(z)=z g(z)
$$

treated as the linear operators on formal series $g(z)$ in the variable $z$. Table 1 lists the $q$-commutators of the combinations of these two operators that we need here and in Section 4.5. The requirement on the 0-th column of $\mathbf{C}_{t}$ reduces to the requirement that on the unit constant function, $\mathrm{x} 1=0, \mathrm{y} 1=z$. When $\sigma=0$, we have $a_{n}(t)=1$, so we are looking for the operators that satisfy

$$
(t \mathrm{x}+\mathrm{y}) z^{n}=z^{n+1}+\text { lower order terms. }
$$

TABLE 1. $q$-commutators $[A, B]_{q}:=A B-q B A$

\begin{tabular}{|r||c|c|c|c|}
\hline${ }^{B} \backslash^{B}$ & $\mathrm{Z}$ & $\mathrm{ZD}_{q}$ & $\mathrm{ZD}_{q}^{2}$ & $\mathrm{Z}^{2} \mathrm{D}_{q}$ \\
\hline \hline $\mathrm{D}_{q}$ & $\mathrm{I}$ & $\mathrm{D}_{q}$ & $\mathrm{D}_{q}^{2}$ & $(1+q) \mathrm{ZD}_{q}-q(1-q) \mathrm{Z}^{2} \mathrm{D}_{q}^{2}$ \\
$\mathrm{ZD}_{q}$ & $\mathrm{Z}$ & $(1-q) \mathrm{ZD}_{q} \mathbf{1}+q(1-q) \mathrm{Z}^{2} \mathrm{D}_{q}^{2}$ & & $\mathrm{Z}^{2} \mathrm{D}_{q}$ \\
$\mathrm{ZD}_{q}^{2}$ & $(1+q) \mathrm{ZD}_{q}-q(1-q) \mathrm{Z}^{2} \mathrm{D}_{q}^{2}$ & $\mathrm{ZD}_{q}^{2}$ & & \\
$\mathrm{Z}^{2} \mathrm{D}_{q}$ & $\mathrm{Z}^{2}$ & & & \\
\hline
\end{tabular}


Example 4.7 ( $q$-Meixner processes). The $q$-Meixner process is a quadratic harness with parameters $\sigma=\eta=0$; see [14]. Inspecting Table 1 we verify that

$$
\mathrm{x}=\mathrm{D}_{q}
$$

and

$$
\mathrm{y}=\mathrm{Z}\left(1+\theta \mathrm{D}_{q}+\tau \mathrm{D}_{q}^{2}\right)
$$

solve (1.1) when $\sigma=\eta=0$. Indeed,

$$
[\mathrm{x}, \mathrm{y}]_{q}=\left[\mathrm{D}_{q}, \mathrm{Z}\right]_{q}+\theta\left[\mathrm{D}_{q}, \mathrm{ZD}_{q}\right]_{q}+\tau\left[\mathrm{D}_{q}, \mathrm{ZD}_{q}^{2}\right]_{q}=\mathrm{I}+\theta \mathrm{D}_{q}+\tau \mathrm{D}_{q}^{2} .
$$

A calculation shows now that

$$
(t \mathrm{x}+\mathrm{y}) z^{n}=z^{n+1}+\theta[n]_{q} z^{n}+\left(t+\tau[n-1]_{q}\right)[n]_{q} z^{n-1},
$$

which by the identification of $z^{n}$ with $p_{n}(x ; t)$ implies that the corresponding martingale polynomials satisfy the three step recurrence

$$
x p_{n}(x ; t)=p_{n+1}(x ; t)+\theta[n]_{q} p_{n}(x ; t)+\left(t+\tau[n-1]_{q}\right)[n]_{q} p_{n-1}(x ; t), n \geq 1 .
$$

Of course, this is a special case of (4.24) corresponding to $\eta=0$. Feinsilver [20, Section 3.4] gives a reparametrization of recurrence (4.32) and considers $q$-commutator relations $[\mathrm{x}, \mathrm{y}]_{q}=h$ as well as their realizations via operators $\mathrm{D}_{q}, \mathrm{Z}$ without considering (1.1). Anshelevich [2, Remark 6] discusses a reparametrization of recurrence (4.32) in relation to free Sheffer systems. In 14 recurrence (4.32) is used as the first step in the construction of the $q$-Meixner Markov processes.

We now use the same method to derive the recurrence for the martingale polynomials of the bi-Poisson process.

Example 4.8 (bi-Poisson process). The bi-Poisson process is a quadratic harness with parameters $\sigma=\tau=0$. Inspecting Table 1 we verify that

$$
\mathrm{x}=\mathrm{D}_{q}+\eta \mathrm{Z}\left(\mathrm{D}_{q}+\theta \mathrm{D}_{q}^{2}\right)
$$

and

$$
\mathrm{y}=\mathrm{Z}\left(1+\theta \mathrm{D}_{q}\right)
$$

solve (1.1) when $\sigma=\tau=0$. Indeed,

$$
\begin{aligned}
{[\mathrm{x}, \mathrm{y}]_{q}=} & {\left[\mathrm{D}_{q}, \mathrm{Z}\right]_{q}+\theta\left[\mathrm{D}_{q}, \mathrm{ZD}_{q}\right]_{q}+\eta\left[\mathrm{ZD}_{q}, \mathrm{Z}\right]_{q}+\eta \theta\left[\mathrm{ZD}_{q}, \mathrm{ZD}_{q}\right]_{q} } \\
& +\eta \theta\left[\mathrm{ZD}_{q}^{2}, \mathrm{Z}\right]_{q}+\eta \theta^{2}\left[\mathrm{ZD}_{q}^{2}, \mathrm{ZD}_{q}\right]_{q} \\
= & \mathrm{I}+\theta \mathrm{D}_{q}+\eta \mathrm{Z}+\eta \theta(1-q) \mathrm{ZD}_{q}+\eta \theta q(1-q) \mathbf{Z}^{2} \mathrm{D}_{q}^{2} \\
& +\eta \theta(1+q) \mathrm{ZD}_{q}-\eta \theta q(1-q) \mathrm{Z}^{2} \mathrm{D}_{q}^{2}+\eta \theta^{2} \mathrm{ZD}_{q}^{2} \\
= & \mathrm{I}+\theta \mathrm{D}_{q}+\eta \mathrm{Z}+\eta \theta \mathrm{ZD}_{q}+\eta \theta \mathrm{ZD}_{q}+\eta \theta^{2} \mathrm{ZD}_{q}^{2} \\
= & \mathrm{I}+\left(\theta \mathrm{D}_{q}+\eta \theta \mathrm{ZD}_{q}+\eta \theta^{2} \mathrm{ZD}_{q}^{2}\right)+\left(\eta \mathrm{Z}+\eta \theta \mathrm{ZD}_{q}\right) \\
= & \mathrm{I}+\theta \mathrm{x}+\eta \mathrm{y} .
\end{aligned}
$$

Operator $t \mathrm{x}+\mathrm{y}$ satisfies the constraint $(t \mathrm{x}+\mathrm{y}) 1=z$. A calculation shows that

$$
(t \mathrm{x}+\mathrm{y}) z^{n}=z^{n+1}+(\theta+t \eta)[n]_{q} z^{n}+t\left(1+\eta \theta[n-1]_{q}\right)[n]_{q} z^{n-1},
$$


so the constraint (4.31) holds. By the identification of $z^{n}$ with $p_{n}(x ; t)$, the corresponding martingale polynomials satisfy the three step recurrence

$$
x p_{n}(x ; t)=p_{n+1}(x ; t)+(\theta+t \eta)[n]_{q} p_{n}(x ; t)+t\left(1+\eta \theta[n-1]_{q}\right)[n]_{q} p_{n-1}(x ; t),
$$

$n \geq 0$, with $p_{-1}=0, p_{0}=1$. Of course, this is a special case of (4.24) corresponding to $\tau=0$. But this recurrence was hard to guess without (1.1), so in 12] it appears for $q=0$ only.

4.5. Dual $q$-commutation equation. Coherent states. Coherent states and the Segal-Bargmann representation are analytical techniques developed in mathematical physics [31. The full Segal-Bargmann isomorphism is known to fail even in the relatively simple case of $q$-Brownian motion with $q<0$; compare [37. But algebraic duality is available and useful in a more general setting. Let

$$
Q_{t, s, u}^{*}(\mathrm{x}, \mathrm{y})=A_{t, s, u} \mathrm{x}^{2}+B_{t, s, u} \mathrm{yx}+C_{t, s, u} \mathrm{y}^{2}+D_{t, s, u} \mathrm{x}+E_{t, s, u} \mathrm{y}+F_{t, s, u}
$$

be the quadratic form in the non-commuting variables $x, y$; for background, see e.g. [25. page 7]. Note that this is a dual of the quadratic form (3.12) that appears in the proof of Theorem 2.3. The following relates (4.34) to the dual version of the $q$-commutation equation (1.1).

Proposition 4.9. Let $\mathrm{X}_{t}$ be a linear function $\mathrm{X}_{t}=\mathrm{x}+$ ty of non-commutative variables $\mathrm{x}, \mathrm{y}$, and suppose that the coefficients of the quadratic form $Q_{t, s, u}^{*}$ are given by (2.15) and (3.6)-(3.10). Then the following statements are equivalent.

(1) The operator identity

$$
\mathrm{X}_{t}^{2}=Q_{t, s, u}^{*}\left(\mathrm{X}_{s}, \mathrm{X}_{u}\right)
$$

holds for all $s<t<u$.

(2) The non-commutative variables $\mathrm{x}, \mathrm{y}$ satisfy the equation

$$
[\mathrm{x}, \mathrm{y}]_{q}=\sigma \mathrm{x}^{2}+\tau \mathrm{y}^{2}+\eta \mathrm{x}+\theta \mathrm{y}+\mathrm{I} .
$$

Proof. Since $\mathrm{X}_{u} \mathrm{X}_{s}=\mathrm{x}^{2}+s u \mathrm{y}^{2}+s(\mathrm{x} \mathrm{y}+\mathrm{yx})+(u-s) \mathrm{yx}$, we have

$$
\begin{aligned}
& \mathrm{X}_{t}^{2}-Q_{t, s, u}^{*}\left(\mathrm{X}_{s}, \mathrm{X}_{u}\right) \\
&=\left(1-A_{t, s, u}-B_{t, s, u}-\right.\left.C_{t, s, u}\right) \mathrm{x}^{2}+\left(t^{2}-s^{2} A_{t, s, u}-s u B_{t, s, u}-u^{2} C_{t, s, u}\right) \mathrm{y}^{2} \\
&+\left(t-s A_{t, s, u}-s B_{t, s, u}-u C_{t, s, u}\right)(\mathrm{xy}+\mathrm{yx})-(u-s) B_{t, s, u} \mathrm{yx} \\
& \quad-\left(D_{t, s, u}+E_{t, s, u}\right) \mathrm{x}-\left(s D_{t, s, u}+u E_{t, s, u}\right) \mathrm{y}-F_{t, s, u} \mathrm{l} .
\end{aligned}
$$

Relations (2.13), (3.4), and (3.5) are equivalent to

$$
\begin{gathered}
\mathrm{X}_{t}^{2}-Q_{t, s, u}^{*}\left(\mathrm{X}_{s}, \mathrm{X}_{u}\right)=-\sigma F_{t, s, u} \mathrm{x}^{2}-\tau F_{t, s, u} \mathrm{y}^{2} \\
+F_{t, s, u}(\mathrm{xy}+\mathrm{yx})-(1+q) F_{t, s, u} \mathrm{yx}-\eta F_{t, s, u} \mathrm{x}-\theta F_{t, s, u} \mathrm{y}-F_{t, s, u} \mathrm{I} \\
=F_{t, s, u}\left([\mathrm{x}, \mathrm{y}]_{q}-\sigma \mathrm{x}^{2}-\tau \mathrm{y}^{2}-\eta \mathrm{x}-\theta \mathrm{y}-\mathrm{I}\right) .
\end{gathered}
$$

4.5.1. Coherent states of $q$-Meixner process. Continuing Example 4.7, the analogue of the coherent state in physics is the generating function

$$
\varphi_{t}(z, x)=\sum_{n=0}^{\infty} \frac{z^{n}}{[n]_{q} !} p_{n}(x ; t)
$$

where $p_{n}(x ; t)$ are the orthogonal martingale polynomials given by recurrence (4.32). 
Proposition 4.10. The operator

$$
\mathbf{X}_{t}=\left(\mathbf{I}+\theta \mathbf{Z}+\tau \mathbf{Z}^{2}\right) \mathbf{D}_{q}+t \mathbf{Z}
$$

satisfies (4.35) with the quadratic form $Q_{t, s, u}^{*}$ given by (2.15), (3.6)-(3.10) where $\sigma=\eta=0$. Moreover,

$$
x \varphi_{t}(z, x)=\left(\mathrm{X}_{t} \varphi_{t}\right)(z, x) .
$$

Proof. Inspecting Table 1 it is easy to verify that $\mathrm{x}=\left(\mathrm{I}+\theta \mathrm{Z}+\tau \mathrm{Z}^{2}\right) \mathrm{D}_{q}$ and $\mathrm{y}=\mathrm{Z}$ satisfy (4.36) with $\sigma=\eta=0$. Indeed,

$$
\begin{aligned}
{[\mathrm{x}, \mathrm{y}]_{q} } & =\left[\mathrm{D}_{q}, \mathrm{Z}\right]_{q}+\theta\left[\mathrm{ZD}_{q}, \mathrm{Z}\right]_{q}+\tau\left[\mathrm{Z}^{2} \mathrm{D}_{q}, \mathrm{Z}\right]_{q} \\
& =\mathrm{I}+\theta \mathbf{Z}+\tau \mathbf{Z}^{2}=\mathrm{I}+\theta \mathbf{y}+\tau \mathrm{y}^{2} .
\end{aligned}
$$

Therefore, Proposition 4.9 implies (4.35). The algebraic identity (4.39) follows from recurrence (4.32) by the following calculation:

$$
\begin{gathered}
x \varphi_{t}(z, x)=\sum_{n=0}^{\infty} \frac{z^{n}}{[n]_{q} !} x p_{n}(x ; t) \\
=\sum_{n=0}^{\infty} \frac{z^{n}}{[n]_{q} !}\left(p_{n+1}(x ; t)+\theta[n] p_{n}(x ; t)+\tau[n][n-1] p_{n-1}(x ; t)+t[n] p_{n-1}(x ; t)\right) \\
=\mathrm{D}_{q} \sum_{n=0}^{\infty} \frac{z^{n+1}}{[n+1]_{q} !} p_{n+1}(x ; t)+\theta z \mathrm{D}_{q} \sum_{n=0}^{\infty} \frac{z^{n}}{[n]_{q} !} p_{n}(x ; t) \\
+\tau z^{2} \mathrm{D}_{q} \sum_{n=1}^{\infty} \frac{z^{n-1}}{[n-1]_{q} !} p_{n-1}(x ; t)+t z \sum_{n=1}^{\infty} \frac{z^{n-1}}{[n-1]_{q} !} p_{n-1}(x ; t) \\
=\left(\mathrm{D}_{q}+\theta \mathrm{ZD}_{q}+\tau \mathrm{Z}^{2} \mathrm{D}_{q}+t \mathrm{Z}\right) \varphi_{t}(z, x) .
\end{gathered}
$$

Formula (4.39) is implicit in the usual derivation of the product formula for $\varphi_{t}(z, x)$; compare [1. When the parameters $\tau, \theta$ vanish, it appears in [37, Section $3]$ in the context of analyzing ground states for the $q$-deformed Gauss distribution. Ref. 3] gives a more general analytical scheme which coincides with (4.38) and (4.39) when $\tau=0, q=1$; however, it advocates the normalization by the $L_{2}$-norm of the polynomials, which does not fit all the cases we are interested in.

In 14 we defined the $q$-Meixner process as a Markov process with the initial state $X_{0}=0$ and with the transition probabilities $P_{s, t}(x, d y)$ determined as the unique probability measure orthogonalizing the polynomials $Q_{n}$ in the variable $y$ which are given by the three step recurrence

$$
\begin{aligned}
y Q_{n}(y \mid x)=Q_{n+1}(y \mid x, t, s) & +\left(\theta[n]_{q}+x q^{n}\right) Q_{n}(y \mid x, t, s) \\
& +\left(t-s q^{n-1}+\tau[n-1]_{q}\right)[n]_{q} Q_{n-1}(y \mid x, t, s) .
\end{aligned}
$$

In that paper, we showed that this Markov process is well defined, that it has orthogonal martingale polynomials $p_{n}(x ; t)$ given by recurrence (4.32), and we used this to prove that $\left(X_{t}\right)$ is a quadratic harness with parameters $\sigma=\eta=0$. Proposition 4.10 simplifies the verification of the quadratic harness condition in 14, Proposition $3.4]$, condensing more than three pages of proof into one page. 
Proposition 4.11 ([14]). If $|q| \leq 1$ and the polynomials $p_{n}(x ; t)$ defined by (4.32) are orthogonal martingale polynomials for a Markov process $\left(X_{t}\right)$, then $\left(X_{t}\right)$ is a quadratic harness with parameters $\sigma=\eta=0$.

Proof. For simplicity, we consider only $|q|<1$, as in this case (4.32) implies that $\left|X_{t}\right| \leq C t$ has bounded support. Consider the generating function (4.37). Since $\left(X_{t}\right)$ is Markov, condition (2.3) is equivalent to

$$
\begin{aligned}
& \mathbb{E}\left(\varphi_{s}\left(z_{1}, X_{s}\right) X_{t} \varphi_{u}\left(z, X_{u}\right)\right) \\
& =a_{t, s, u} \mathbb{E}\left(\varphi_{s}\left(z_{1}, X_{s}\right) X_{s} \varphi_{s}\left(z, X_{s}\right)\right)+b_{t, s, u} \mathbb{E}\left(\varphi_{s}\left(z_{1}, X_{s}\right) X_{u} \varphi_{u}\left(z, X_{u}\right)\right)
\end{aligned}
$$

holding for all $z_{1}, z$ in a neighborhood of 0 (or just as an identity in formal power series in the variables $z_{1}, z$ ). We now use the martingale polynomial property, which implies that

$$
\mathbb{E}\left[\varphi_{t}\left(z, X_{t}\right) \mid X_{s}\right]=\varphi_{s}\left(z, X_{s}\right),
$$

and we use (4.39) to represent the process through the operator (4.38) as

$$
X_{t} \varphi_{t}\left(z, X_{t}\right)=\mathrm{X}_{t}\left(\varphi_{t}\left(z, X_{t}\right)\right) \text {. }
$$

This gives

$$
\begin{aligned}
\mathbb{E}\left(\varphi_{s}\left(z_{1}, X_{s}\right) X_{t} \varphi_{u}\left(z, X_{u}\right)\right) & =\mathbb{E}\left(\varphi_{s}\left(z_{1}, X_{s}\right) \mathrm{X}_{t} \varphi_{t}\left(z, X_{t}\right)\right) \\
& =\mathrm{X}_{t} \mathbb{E}\left(\varphi_{s}\left(z_{1}, X_{s}\right) \varphi_{s}\left(z, X_{s}\right)\right)=\mathrm{X}_{t} G_{s}\left(z_{1}, z\right),
\end{aligned}
$$

where

$$
\begin{aligned}
G_{s}\left(z_{1}, z\right)=\mathbb{E}\left(\varphi_{s}\left(z_{1}, X_{s}\right) \varphi_{s}\left(z, X_{s}\right)\right) & =\sum_{n=0}^{\infty} \frac{\left(z_{1} z\right)^{n}}{[n]_{q} !^{2}} \mathbb{E}\left(p_{n}\left(X_{s} ; s\right)^{2}\right) \\
& =\sum_{n=0}^{\infty} \frac{\left(z_{1} z\right)^{n}}{[n]_{q} !} \prod_{k=1}^{n}(t+\tau[k-1]),
\end{aligned}
$$

and $\mathrm{X}_{t}$ acts on $G_{s}\left(z_{1}, z\right)$ as a series in the variable $z$. Therefore, equation (4.41) is equivalent to

$$
\mathrm{X}_{t} G_{s}\left(z_{1}, z\right)=a_{t, s, u} \mathrm{X}_{s} G_{s}\left(z_{1}, z\right)+b_{t, s, u} \mathrm{X}_{u} G_{s}\left(z_{1}, z\right)
$$

and follows from the (trivial) operator identity $\mathbf{X}_{t}=a_{t, s, u} \mathbf{X}_{s}+b_{t, s, u} \mathbf{X}_{u}$. Similarly, condition (2.11) is equivalent to

$$
\begin{aligned}
& \mathbb{E}\left(\varphi_{s}\left(z_{1}, X_{s}\right) X_{t}^{2} \varphi_{u}\left(z, X_{u}\right)\right)=A_{t, s, u} \mathbb{E}\left(\varphi_{s}\left(z_{1}, X_{s}\right) X_{s}^{2} \varphi_{s}\left(z, X_{s}\right)\right) \\
& +B_{t, s, u} \mathbb{E}\left(\varphi_{s}\left(z_{1}, X_{s}\right) X_{s} X_{u} \varphi_{u}\left(z, X_{u}\right)\right)+C_{t, s, u} \mathbb{E}\left(\varphi_{s}\left(z_{1}, X_{s}\right) X_{u}^{2} \varphi_{u}\left(z, X_{u}\right)\right) \\
& +D_{t, s, u} \mathbb{E}\left(\varphi_{s}\left(z_{1}, X_{s}\right) X_{s} \varphi_{s}\left(z, X_{s}\right)\right)+E_{t, s, u} \mathbb{E}\left(\varphi_{s}\left(z_{1}, X_{s}\right) X_{u} \varphi_{u}\left(z, X_{u}\right)\right) \\
& +F_{t, s, u} \mathbb{E}\left(\varphi_{s}\left(z_{1}, X_{s}\right) \varphi_{s}\left(z, X_{s}\right)\right) .
\end{aligned}
$$

Notice that for $s \leq u$ we have

$$
\begin{gathered}
\mathbb{E}\left(\varphi_{s}\left(z_{1}, X_{s}\right) X_{s} X_{u} \varphi_{u}\left(z, X_{u}\right)\right)=\mathbb{E}\left(\varphi_{s}\left(z_{1}, X_{s}\right) X_{s} \mathrm{X}_{u} \varphi_{u}\left(z, X_{u}\right)\right) \\
=\mathrm{X}_{u} \mathbb{E}\left(\varphi_{s}\left(z_{1}, X_{s}\right) X_{s} \varphi_{s}\left(z, X_{s}\right)\right)=\mathrm{X}_{u} \mathrm{X}_{s} \mathbb{E}\left(\varphi_{s}\left(z_{1}, X_{s}\right) \varphi_{s}\left(z, X_{s}\right)\right)=\mathrm{X}_{u} \mathrm{X}_{s} G_{s}\left(z_{1}, z\right) .
\end{gathered}
$$

Therefore, equation (4.43) follows from the operator identity (4.35), which in expanded form says

$$
\mathrm{X}_{t}^{2}=A_{t, s, u} \mathrm{X}_{s}^{2}+B_{t, s, u} \mathrm{X}_{u} \mathrm{X}_{s}+C_{t, s, u} \mathrm{X}_{u}^{2}+D_{t, s, u} \mathrm{X}_{s}+E_{t, s, u} \mathrm{X}_{u}+F_{t, s, u} \mathrm{l}
$$

applied to $G_{s}\left(z_{1}, z\right)$ treated as a series in the variable $z$. 
4.5.2. Coherent states of the bi-Poisson process. We now repeat the methods of Section 4.5.1 to derive new results about the bi-Poisson process from Example 4.8.

Proposition 4.12. The operator

$$
\mathbf{X}_{t}=\left(\mathrm{I}+(\theta+\eta t) \mathbf{Z}+t \eta \theta \mathbf{Z}^{2}\right) \mathrm{D}_{q}+t \mathbf{Z}
$$

satisfies (4.35) for the quadratic form $Q_{t, s, u}^{*}$ with the coefficients (2.15), (3.6)(3.10) such that $\sigma=\tau=0$. Moreover, if $\varphi_{t}(z, x)$ is the generating function (4.37) of the orthogonal martingale polynomials $p_{n}(x ; t)$ given by recurrence (4.33), then

$$
x \varphi_{t}(z, x)=\left(\mathrm{X}_{t} \varphi_{t}\right)(z, x) \text {. }
$$

Proof. Inspecting Table 1, we verify that

$$
\mathrm{x}=(\mathrm{I}+\theta \mathrm{Z}) \mathrm{D}_{q}
$$

and

$$
\mathrm{y}=\mathrm{Z}+\eta\left(\mathrm{Z}+\theta \mathrm{Z}^{2}\right) \mathrm{D}_{q}
$$

solve (4.36) with $\sigma=\tau=0$. Indeed,

$$
\begin{aligned}
{[\mathrm{x}, \mathrm{y}]_{q} } & =\left[\mathrm{D}_{q}, \mathrm{Z}\right]_{q}+\theta\left[\mathrm{ZD}_{q}, \mathrm{Z}\right]_{q}+\eta\left[\mathrm{D}_{q}, \mathrm{ZD}_{q}\right]_{q}+\eta \theta\left[\mathrm{ZD}_{q}, \mathrm{ZD}_{q}\right]_{q}+\eta \theta\left[\mathrm{D}_{q}, \mathrm{Z}^{2} \mathrm{D}_{q}\right]_{q} \\
& +\eta \theta^{2}\left[\mathrm{ZD}_{q}, \mathrm{Z}^{2} \mathrm{D}_{q}\right]_{q}=\mathrm{I}+\theta \mathrm{Z}+\eta \mathrm{D}_{q}+\eta \theta\left((1-q) \mathrm{ZD}_{q}+q(1-q) \mathrm{Z}^{2} \mathrm{D}_{q}^{2}\right) \\
& +\eta \theta\left((1+q) \mathrm{ZD}_{q}-q(1-q) \mathrm{Z}^{2} \mathrm{D}_{q}^{2}\right)+\eta \theta^{2} \mathrm{Z}^{2} \mathrm{D}_{q}=\mathrm{I}+\theta \mathrm{Z}+\eta \mathrm{D}_{q}+\eta \theta \mathrm{Z} \mathrm{D}_{q} \\
& +\eta \theta \mathrm{ZD}_{q}+\eta \theta^{2} \mathrm{Z}^{2} \mathrm{D}_{q}=\mathrm{I}+\eta(\mathrm{I}+\theta \mathrm{Z}) \mathrm{D}_{q}+\theta\left(\mathrm{Z}+\eta\left(\mathrm{Z} \theta \mathrm{Z}^{2}\right) \mathrm{D}_{q}\right)=\mathrm{I}+\eta \mathrm{x}+\theta \mathrm{y} .
\end{aligned}
$$

Therefore, Proposition 4.9 implies (4.35). We now derive (4.45) from (4.33) by the following calculation:

$$
\begin{gathered}
x \varphi_{t}(z, x)=\sum_{n=0}^{\infty} \frac{z^{n}}{[n]_{q} !} x p_{n}(x ; t) \\
=\sum_{n=0}^{\infty} \frac{z^{n}}{[n]_{q} !}\left(p_{n+1}(x ; t)+(\theta+t \eta)[n]_{q} p_{n}(x ; t)+t\left(1+\eta \theta[n-1]_{q}\right)[n]_{q} p_{n-1}(x ; t)\right) \\
=\mathrm{D}_{q} \sum_{n=0}^{\infty} \frac{z^{n+1}}{[n+1]_{q} !} p_{n+1}(x ; t)+(\theta+t \eta) z \mathrm{D}_{q} \sum_{n=0}^{\infty} \frac{z^{n}}{[n]_{q} !} p_{n}(x ; t) \\
+t z \sum_{n=1}^{\infty} \frac{z^{n-1}}{[n-1]_{q} !} p_{n-1}(x ; t)+t \eta \theta z^{2} \mathrm{D}_{q} \sum_{n=1}^{\infty} \frac{z^{n-1}}{[n-1]_{q} !} p_{n-1}(x ; t) \\
=\left(\left(\mathrm{I}+(\theta+\eta t) \mathrm{Z}+t \eta \theta \mathrm{Z}^{2}\right) \mathrm{D}_{q}+t \mathrm{Z}\right) \varphi_{t}(z, x) .
\end{gathered}
$$

Next we give the bi-Poisson version of Proposition 4.11.

Proposition 4.13. Fix $-1 \leq q \leq 1$. If polynomials $p_{n}(x ; t)$ given by (4.33) are orthogonal martingale polynomials for a Markov process $\left(X_{t}\right)$, then $\left(X_{t}\right)$ is a biPoisson process, i.e., (2.3) and (2.14) hold with $\sigma=\tau=0$, and $1+\eta \theta \geq \max \{q, 0\}$. Moreover, such a Markov process $\left(X_{t}\right)$ is determined uniquely.

Proof. Property (2.6) follows from the explicit form of the polynomials $p_{0}, p_{1}, p_{2}$. Orthogonality gives $\mathbb{E}\left(X_{t}\right)=\mathbb{E}\left(p_{1}\left(X_{t} ; t\right) p_{0}\left(X_{t} ; t\right)\right)=0$ and $\mathbb{E}\left(X_{t}^{2}\right)=\mathbb{E}\left(\left(p_{2}\left(X_{t} ; t\right)+\right.\right.$ $\left.\left.(\theta+\eta t) X_{t}+t\right) p_{0}\left(X_{t}\right)\right)=t$. The martingale polynomial property then implies that $\mathbb{E}\left(X_{s} X_{t}\right)=\mathbb{E}\left(X_{s} \mathbb{E}\left[p_{1}\left(X_{t} ; t\right) \mid \mathcal{F}_{\leq s}\right]\right)=\mathbb{E}\left(X_{s}^{2}\right)=s$. 
If $-1 \leq q<1$, from (4.33) it follows that $\left|X_{t}\right| \leq C t$ has bounded support; if $q=1$, then it is not hard to identify the distribution of $\left|X_{t}\right|$ for example, from [35. Chapter 4] or [15, pages 175-181], verifying that $\left|X_{t}\right|$ has a finite exponential moment. Thus the moment problem has a unique solution and the process $\left(X_{t}\right)$ is determined uniquely. Moreover, polynomials are dense in $L_{2}\left(X_{s}, X_{u}\right)$; see [18, Theorem 3.1.18]. Consider the generating function (4.37) with polynomials $p_{n}(x ; t)$ satisfying (4.33). Notice that property (4.42) follows again from the martingale polynomial condition. By Proposition 4.12 with $\mathrm{X}_{t}$ defined by (4.44) we have the representation

$$
X_{t} \varphi_{t}\left(z, X_{t}\right)=\mathrm{X}_{t}\left(\varphi_{t}\left(z, X_{t}\right)\right)
$$

Since $\left(X_{t}\right)$ is Markov and polynomials are dense in $L_{2}\left(X_{s}, X_{u}\right)$, condition (2.3) is again equivalent to (4.41), which we can interpret as the identity between the formal power series in the variables $z_{1}, z$. The latter follows again from the (trivial) operator identity $\mathbf{X}_{t}=a_{t, s, u} \mathbf{X}_{s}+b_{t, s, u} \mathbf{X}_{u}$, applied to

$$
G_{s}\left(z_{1}, z\right)=\mathbb{E}\left(\varphi_{s}\left(z_{1}, X_{s}\right) \varphi_{s}\left(z, X_{s}\right)\right)=\sum_{n=0}^{\infty} \frac{\left(z_{1} z\right)^{n}}{[n]_{q} !^{2}} \mathbb{E}\left(p_{n}\left(X_{s} ; s\right)^{2}\right),
$$

treated as the formal power series in the variable $z$. Similarly, condition (2.11) is equivalent to (4.43), and again

$$
\mathbb{E}\left(\varphi_{s}\left(z_{1}, X_{s}\right) X_{s} X_{u} \varphi_{u}\left(z, X_{u}\right)\right)=\mathrm{X}_{u} \mathrm{X}_{s} G_{s}\left(z_{1}, z\right) .
$$

Therefore, equation (4.43) follows from the operator identity (4.35) applied to $G_{s}\left(z_{1}, z\right)$ treated as the formal power series in the variable $z$. Since the third coefficient in (4.33) is nonnegative for all $n$, we get $1+\eta \theta \geq \max \{q, 0\}$; compare Remark 4.5

\section{Appendix A. Proofs of Claims and lemmas}

Throughout the proofs of the next three Claims, $0<r<s<t<u$ are arbitrary numbers.

Proof of Claim 3.1. Observe that from (2.3) and (2.11) we get

$$
\begin{gathered}
\mathbb{E}\left[X_{s} X_{t} \mid \mathcal{F}_{r, u}\right]=\mathbb{E}\left[X_{s} \mathbb{E}\left[X_{t} \mid \mathcal{F}_{s, u}\right] \mid \mathcal{F}_{r, u}\right] \\
=a_{t, s, u} \mathbb{E}\left[X_{s}^{2} \mid \mathcal{F}_{r, u}\right]+b_{t, s, u} X_{u} \mathbb{E}\left[X_{s} \mid \mathcal{F}_{r, u}\right] \\
=a_{t, s, u}\left(A_{s, r, u} X_{r}^{2}+B_{s, r, u} X_{r} X_{u}+C_{s, r, u} X_{u}^{2}+D_{s, r, u} X_{r}+E_{s, r, u} X_{u}+F_{s, r, u}\right) \\
+b_{t, s, u} X_{u}\left(a_{s, r, u} X_{r}+b_{s, r, u} X_{u}\right)=a_{t, s, u} A_{s, r, u} X_{r}^{2}+\left(a_{t, s, u} B_{s, r, u}+b_{t, s, u} a_{s, r, u}\right) X_{r} X_{u} \\
+\left(a_{t, s, u} C_{s, r, u}+b_{t, s, u} b_{s, r, u}\right) X_{u}^{2}+a_{t, s, u} D_{s, r, u} X_{r}+a_{t, s, u} E_{s, r, u} X_{u}+a_{t, s, u} F_{s, r, u} .
\end{gathered}
$$

On the other hand,

$$
\begin{gathered}
\mathbb{E}\left[X_{s} X_{t} \mid \mathcal{F}_{r, u}\right]=\mathbb{E}\left[\mathbb{E}\left[X_{s} \mid \mathcal{F}_{r, t}\right] X_{t} \mid \mathcal{F}_{r, u}\right]=a_{s, r, t} X_{r} \mathbb{E}\left[X_{t} \mid \mathcal{F}_{r, u}\right]+b_{s, r, t} \mathbb{E}\left[X_{t}^{2} \mid \mathcal{F}_{r, u}\right] \\
=a_{s, r, t} X_{r}\left(a_{t, r, u} X_{r}+b_{t, r, u} X_{u}\right) \\
+b_{s, r, t}\left(A_{t, r, u} X_{r}^{2}+B_{t, r, u} X_{r} X_{u}+C_{t, r, u} X_{u}^{2}+D_{t, r, u} X_{r}+E_{t, r, u} X_{u}+F_{t, r, u}\right) \\
=\left(a_{s, r, t} a_{t, r, u}+b_{s, r, t} A_{t, r, u}\right) X_{r}^{2}+\left(a_{s, r, t} b_{t, r, u}+b_{s, r, t} B_{t, r, u}\right) X_{r} X_{u} \\
+b_{s, r, t} C_{t, r, u} X_{u}^{2}+b_{s, r, t} D_{t, r, u} X_{r}+b_{s, r, t} E_{t, r, u} X_{u}+b_{s, r, t} F_{t, r, u}
\end{gathered}
$$


Comparing the coefficients at $X_{r}^{2}, X_{r} X_{u}, X_{u}^{2}, X_{r}, X_{u}$ and 1 in the above expressions we get

$$
\begin{aligned}
a_{t, s, u} A_{s, r, u} & =b_{s, r, t} A_{t, r, u}+a_{s, r, t} a_{t, r, u}, \\
a_{t, s, u} B_{s, r, u} & =b_{s, r, t} B_{t, r, u}, \\
b_{t, s, u} b_{s, r, u}+a_{t, s, u} C_{s, r, u} & =b_{s, r, t} C_{t, r, u}, \\
a_{t, s, u} D_{s, r, u} & =b_{s, r, t} D_{t, r, u}, \\
a_{t, s, u} E_{s, r, u} & =b_{s, r, t} E_{t, r, u}, \\
a_{t, s, u} F_{s, r, u} & =b_{s, r, t} F_{t, r, u} .
\end{aligned}
$$

( In the derivation of (A.2) we used the fact that $b_{t, s, u} a_{s, r, u}=a_{s, r, t} b_{t, r, u}$.) Adding (A.1), A.2) and (A.3), we get

$$
\begin{gathered}
a_{t, s, u}\left(A_{s, r, u}+B_{s, r, u}+C_{s, r, u}-1\right)+a_{t, s, u}+b_{t, s, u} b_{s, r, u} \\
=b_{s, r, t}\left(A_{t, r, u}+B_{t, r, u}+C_{t, r, u}-1\right)+b_{s, r, t}+a_{s, r, t} a_{t, r, u} .
\end{gathered}
$$

Since a calculation shows that

$$
a_{t, s, u}+b_{t, s, u} b_{s, r, u}=b_{s, r, t}+a_{s, r, t} a_{t, r, u},
$$

dividing by (A.6), we get $\sigma_{s, r, u}=\sigma_{t, r, u}$, which proves (3.1) when $f=\sigma$. We proceed similarly when $f=\tau, q, \eta, \theta$. Adding (A.1) multiplied by $r^{2}$, (A.2) multiplied by $r u$, (A.3) multiplied by $u^{2}$, and dividing by (A.6), we obtain $\tau_{s, r, u}=\tau_{t, r, u}$, after noticing that $s^{2} a_{t, s, u}+u^{2} b_{t, s, u} b_{s, r, u}=t^{2} b_{s, r, t}+r^{2} a_{s, r, t} a_{t, r, u}$. Equation (A.2) multiplied by $(u-r)$ and divided by (A.6) gives $q_{s, r, u}=q_{t, r, u}$. Adding equations (A.4) and (A.5) and dividing by (A.6) gives $\eta_{s, r, u}=\eta_{t, r, u}$ and similarly multiplying (A.4) by $r$ and (A.5) by $u$ after dividing by (A.6) gives $\theta_{s, r, u}=\theta_{t, r, u}$. Thus we have obtained $f_{s, r, u}=f_{t, r, u}$ for $f=\sigma, \tau, q, \eta, \theta$. If $0<x<y<z$, then substitution $r=x, s=y, u=z$ yields (3.1) for $\widetilde{y} \in(y, z)$; substitution $r=x, t=y, u=z$ gives (3.1) for $\widetilde{y} \in(x, y)$, completing the proof of Claim (3.1).

Proof of Claim 3.2, Now consider the identity

$$
\mathbb{E}\left[X_{t}^{2} \mid \mathcal{F}_{r, u}\right]=\mathbb{E}\left[\mathbb{E}\left[X_{t}^{2} \mid \mathcal{F}_{s, u}\right] \mid \mathcal{F}_{r, u}\right] .
$$

Using (2.3) and (2.11) we see that $\mathbb{E}\left[\mathbb{E}\left[X_{t}^{2} \mid \mathcal{F}_{s, u}\right] \mid \mathcal{F}_{r, u}\right]$ is given by

$$
\begin{gathered}
\mathbb{E}\left[A_{t, s, u} X_{s}^{2}+B_{t, s, u} X_{s} X_{u}+C_{t, s, u} X_{u}^{2}+D_{t, s, u} X_{s}+E_{t, s, u} X_{u}+F_{t, s, u} \mid \mathcal{F}_{r, u}\right] \\
=A_{t, s, u} \mathbb{E}\left[X_{s}^{2} \mid \mathcal{F}_{r, u}\right]+B_{t, s, u} X_{u} \mathbb{E}\left[X_{s} \mid \mathcal{F}_{r, u}\right]+C_{t, s, u} X_{u}^{2}+D_{t, s, u} \mathbb{E}\left[X_{s} \mid \mathcal{F}_{r, u}\right] \\
+E_{t, s, u} X_{u}+F_{t, s, u}=A_{t, s, u}\left(A_{s, r, u} X_{r}^{2}+B_{s, r, u} X_{r} X_{u}+C_{s, r, u} X_{u}^{2}+D_{s, r, u} X_{r}\right. \\
\left.+E_{s, r, u} X_{u}+F_{s, r, u}\right)+B_{t, s, u} X_{u}\left(a_{s, r, u} X_{r}+b_{s, r, u} X_{u}\right)+C_{t, s, u} X_{u}^{2} \\
+D_{t, s, u}\left(a_{s, r, u} X_{r}+b_{s, r, u} X_{u}\right)+E_{t, s, u} X_{u}+F_{t, s, u}=A_{t, s, u} A_{s, r, u} X_{r}^{2} \\
+\left(A_{t, s, u} B_{s, r, u}+B_{t, s, u} a_{s, r, u}\right) X_{r} X_{u}+\left(A_{t, s, u} C_{s, r, u}+B_{t, s, u} b_{s, r, u}+C_{t, s, u}\right) X_{u}^{2} \\
+\left(A_{t, s, u} D_{s, r, u}+D_{t, s, u} a_{s, r, u}\right) X_{r}+\left(A_{t, s, u} E_{s, r, u}+D_{t, s, u} b_{s, r, u}+E_{t, s, u}\right) X_{u} \\
+A_{t, s, u} F_{s, r, u}+F_{t, s, u} .
\end{gathered}
$$

On the other hand,

$$
\mathbb{E}\left[X_{t}^{2} \mid \mathcal{F}_{r, u}\right]=A_{t, r, u} X_{r}^{2}+B_{t, r, u} X_{r} X_{u}+C_{t, r, u} X_{u}^{2}+C_{t, r, u} X_{r}+D_{t, r, u} X_{u}+F_{t, r, u} .
$$


Comparing the coefficients at $X_{r}^{2}, X_{r} X_{u}, X_{u}^{2}, X_{r}, X_{u}$ and 1, we obtain

$$
\begin{aligned}
A_{t, r, u} & =A_{t, s, u} A_{s, r, u}, \\
B_{t, r, u} & =A_{t, s, u} B_{s, r, u}+B_{t, s, u} a_{s, r, u}, \\
C_{t, r, u} & =A_{t, s, u} C_{s, r, u}+B_{t, s, u} b_{s, r, u}+C_{t, s, u}, \\
D_{t, r, u} & =A_{t, s, u} D_{s, r, u}+D_{t, s, u} a_{s, r, u}, \\
E_{t, r, u} & =A_{t, s, u} E_{s, r, u}+D_{t, s, u} b_{s, r, u}+E_{t, s, u}, \\
F_{t, r, u} & =A_{t, s, u} F_{s, r, u}+F_{t, s, u} .
\end{aligned}
$$

Substituting the right-hand sides of equation A.8 (A.13) for $A_{t, r, u}, B_{t, r, u}, \ldots$, $F_{t, r, u}$ on the right-hand sides of (A.1) - A.6) we get

$$
\begin{aligned}
& A_{s, r, u}\left(a_{t, s, u}-b_{s, r, t} A_{t, s, u}\right)=a_{s, r, t} a_{t, r, u} \\
& B_{s, r, u}\left(a_{t, s, u}-b_{s, r, t} A_{t, s, u}\right)=b_{s, r, t} B_{t, s, u} a_{s, r, u} \\
& C_{s, r, u}\left(a_{t, s, u}-b_{s, r, t} A_{t, s, u}\right)=b_{s, r, t}\left(B_{t, s, u} b_{s, r, u}+C_{t, s, u}\right)-b_{t, s, u} b_{s, r, u}, \\
& D_{s, r, u}\left(a_{t, s, u}-b_{s, r, t} A_{t, s, u}\right)=b_{s, r, t} D_{t, s, u} a_{s, r, u} \\
& E_{s, r, u}\left(a_{t, s, u}-b_{s, r, t} A_{t, s, u}\right)=b_{s, r, t}\left(D_{t, s, u} b_{s, r, u}+E_{t, s, u}\right) \\
& F_{s, r, u}\left(a_{t, s, u}-b_{s, r, t} A_{t, s, u}\right)=b_{s, r, t} F_{t, s, u} .
\end{aligned}
$$

We can now proceed analogously to the proof of Claim 3.1. Namely, adding (A.14), (A.15), A.16), again taking into account A.7), and dividing by (A.19), we get $\sigma_{s, r, u}=\sigma_{t, s, u}$. Adding (A.14) multiplied by $r^{2}$, A.15) multiplied by $r u$ and (A.16) multiplied by $u^{2}$, and dividing by (A.19), we obtain $\tau_{s, r, u}=\tau_{t, s, u}$. Equation (A.15) multiplied by $(u-r)$ and divided by (A.19) gives $q_{s, r, u}=q_{t, s, u}$. Adding equations (A.17) and (A.18) and dividing by (A.19) gives $\eta_{s, r, u}=\eta_{t, s, u}$ and similarly multiplying (A.17) by $r$ and (A.18) by $u$ after dividing by (A.19) gives $\theta_{s, r, u}=\theta_{t, s, u}$. Thus we have obtained $f_{s, r, u}=f_{t, s, u}$ for $f=\sigma, \tau, q, \eta, \theta$. By Claim $3.1 f_{s, r, u}=f_{t, r, u}$, so $f_{t, r, u}=f_{t, s, u}$, ending the proof of Claim 3.2.

Proof of Claim 3.3. This follows from Claim 3.2 by the time-inversion $\left(X_{t}\right) \mapsto$ $\left(t X_{1 / t}\right)$. Alternatively, one can repeat the previous arguments, starting with the identity

$$
\mathbb{E}\left[X_{s}^{2} \mid \mathcal{F}_{r, u}\right]=\mathbb{E}\left[\mathbb{E}\left[X_{s}^{2} \mid \mathcal{F}_{r, t}\right] \mid \mathcal{F}_{r, u}\right] .
$$

Proof of Lemma 3.4, For a polynomial $\varphi: \mathbb{R} \rightarrow \mathbb{R}$, consider the vectors

$$
\mathbf{v}_{s, t}=\left[\mathbb{E}\left(\varphi\left(X_{s}\right) p_{0}\left(X_{t} ; t\right)\right), \mathbb{E}\left(\varphi\left(X_{s}\right) p_{1}\left(X_{t} ; t\right)\right), \ldots, \mathbb{E}\left(\varphi\left(X_{s}\right) p_{k}\left(X_{t} ; t\right)\right), \ldots\right] \in \mathbb{R}^{\infty} .
$$

Since for $s>0$ the random variable $X_{s}$ has infinite support, then the polynomials $1, X_{s}, X_{s}^{2}, \ldots$ are linearly independent, and the corresponding orthogonal polynomials are non-degenerate. This implies that as we change $\varphi$ for a fixed $s>0$, vectors of the form $\mathbf{v}_{s, s}$ are dense in $\mathbb{R}^{\infty}$, equipped with the product topology. Indeed, applying the Gram-Schmidt orthogonalization process to $p_{0}(x ; s), p_{1}(x ; s), \ldots$ we get a sequence of orthogonal polynomials $q_{0}, q_{1}, \ldots$ such that $\mathbb{E}\left(q_{k}\left(X_{s}\right)^{2}\right) \neq 0$. Therefore, for any $\mathbf{w}=\left[w_{0}, w_{1}, \ldots\right] \in \mathbb{R}^{\infty}$ and $n \geq 0$, we can find numbers $u_{0}, u_{1}, \ldots, u_{n}$ such that the first $n+1$ coordinates of $\mathbf{v}_{s, s}$ corresponding to $\varphi=\sum_{j=0}^{n} u_{j} q_{j}$ are equal to $w_{0}, w_{1}, \ldots, w_{n}$. This implies that to verify the identities, it suffices to verify that the identities hold true when multiplied from the left by $\mathbf{v}_{s, s}$. 
By the martingale polynomial property, $\mathbb{E}\left(\varphi\left(X_{s}\right) p_{k}\left(X_{s} ; s\right)\right)=\mathbb{E}\left(\varphi\left(X_{s}\right) p_{k}\left(X_{t} ; t\right)\right)$. Therefore, from (2.17) it follows that $\mathbf{v}_{s, s}=\mathbf{v}_{s, t}$ and

$$
\begin{gathered}
\mathbf{v}_{s, s} \times \mathbf{C}_{t}=\mathbf{v}_{s, t} \times \mathbf{C}_{t} \\
=\left[\mathbb{E}\left(\varphi\left(X_{s}\right) \sum_{j} C_{j, 0}(t) p_{j}\left(X_{t} ; t\right)\right), \mathbb{E}\left(\varphi\left(X_{s}\right) \sum_{j} C_{j, 1}(t) p_{j}\left(X_{t} ; t\right)\right), \ldots\right] \\
=\left[\mathbb{E}\left(\varphi\left(X_{s}\right) X_{t} p_{0}\left(X_{t} ; t\right)\right), \mathbb{E}\left(\varphi\left(X_{s}\right) X_{t} p_{1}\left(X_{t} ; t\right)\right), \ldots, \mathbb{E}\left(\varphi\left(X_{s}\right) X_{t} p_{k}\left(X_{t} ; t\right)\right), \ldots\right] \\
=\left[\mathbb{E}\left(\varphi\left(X_{s}\right) X_{t} p_{0}\left(X_{u} ; u\right)\right), \mathbb{E}\left(\varphi\left(X_{s}\right) X_{t} p_{1}\left(X_{u} ; u\right)\right), \ldots, \mathbb{E}\left(\varphi\left(X_{s}\right) X_{t} p_{k}\left(X_{u} ; u\right)\right), \ldots\right] \\
=a_{t, s, u}\left[\mathbb{E}\left(\varphi\left(X_{s}\right) X_{s} p_{k}\left(X_{u} ; u\right)\right): k \geq 0\right]+b_{t, s, u}\left[\mathbb{E}\left(\varphi\left(X_{s}\right) X_{u} p_{k}\left(X_{u} ; u\right)\right): k \geq 0\right] .
\end{gathered}
$$

Using the martingale polynomial property again, we see that $\mathbf{v}_{s, s} \times \mathbf{C}_{t}$ is equal to

$$
\begin{gathered}
a_{t, s, u}\left[\mathbb{E}\left(\varphi\left(X_{s}\right) X_{s} p_{k}\left(X_{s} ; s\right)\right): k \geq 0\right]+b_{t, s, u}\left[\mathbb{E}\left(\varphi\left(X_{s}\right) X_{u} p_{k}\left(X_{u} ; u\right)\right): k \geq 0\right] \\
=a_{t, s, u} \mathbf{v}_{s, s} \times \mathbf{C}_{s}+b_{t, s, u} \mathbf{v}_{s, u} \times \mathbf{C}_{u}=\mathbf{v}_{s, s} \times\left(a_{t, s, u} \mathbf{C}_{s}+b_{t, s, u} \mathbf{C}_{u}\right),
\end{gathered}
$$

proving (3.11). Similar reasoning proves (3.12):

$$
\begin{aligned}
& \mathbf{v}_{s, s} \times \mathbf{C}_{t}^{2}=\mathbf{v}_{s, t} \times \mathbf{C}_{t}^{2}=\left[\mathbb{E}\left(\varphi\left(X_{s}\right) X_{t} p_{k}\left(X_{t} ; t\right)\right): k \geq 0\right] \times \mathbf{C}_{t} \\
= & {\left[\mathbb{E}\left(\varphi\left(X_{s}\right) X_{t}^{2} p_{k}\left(X_{t} ; t\right)\right): k \geq 0\right]=\left[\mathbb{E}\left(\varphi\left(X_{s}\right) X_{t}^{2} p_{k}\left(X_{u} ; u\right)\right): k \geq 0\right] } \\
= & {\left[\mathbb{E}\left(\varphi\left(X_{s}\right) \mathbb{E}\left[X_{t}^{2} \mid \mathcal{F}_{s, u}\right] p_{k}\left(X_{u} ; u\right)\right): k \geq 0\right] } \\
= & {\left[\mathbb{E}\left(\varphi\left(X_{s}\right) Q_{t, s, u}\left(X_{s}, X_{t}\right) p_{k}\left(X_{u} ; u\right)\right): k \geq 0\right] } \\
= & A_{t, s, u}\left[\mathbb{E}\left(\varphi\left(X_{s}\right) X_{s}^{2} p_{k}\left(X_{s} ; s\right)\right): k \geq 0\right]+B_{t, s, u}\left[\mathbb{E}\left(\varphi\left(X_{s}\right) X_{s} X_{u} p_{k}\left(X_{u} ; u\right)\right): k \geq 0\right] \\
& +C_{t, s, u}\left[\mathbb{E}\left(\varphi\left(X_{s}\right) X_{u}^{2} p_{k}\left(X_{u} ; u\right)\right): k \geq 0\right]+D_{t, s, u}\left[\mathbb{E}\left(\varphi\left(X_{s}\right) X_{s} p_{k}\left(X_{s} ; s\right)\right): k \geq 0\right] \\
& +E_{t, s, u}\left[\mathbb{E}\left(\varphi\left(X_{s}\right) X_{u} p_{k}\left(X_{u} ; u\right)\right): k \geq 0\right]+F_{t, s, u}\left[\mathbb{E}\left(\varphi\left(X_{s}\right) p_{k}\left(X_{s} ; s\right)\right): k \geq 0\right] \\
= & A_{t, s, u} \mathbf{v}_{s, s} \times \mathbf{C}_{s}^{2}+B_{t, s, u}\left[\mathbb{E}\left(\varphi\left(X_{s}\right) X_{s} p_{k}\left(X_{s} ; s\right)\right): k \geq 0\right] \times \mathbf{C}_{u} \\
& +C_{t, s, u} \mathbf{v}_{s, s} \times \mathbf{C}_{u}^{2}+D_{t, s, u} \mathbf{v}_{s, s} \times \mathbf{C}_{s}+E_{t, s, u} \mathbf{v}_{s, s} \times \mathbf{C}_{u}+F_{t, s, u} \mathbf{v}_{s, s} \\
= & \mathbf{v}_{s, s} \times\left(A_{t, s, u} \mathbf{C}_{s}^{2}+B_{t, s, u} \mathbf{C}_{s} \mathbf{C}_{u}+C_{t, s, u} \mathbf{C}_{u}^{2}+D_{t, s, u} \mathbf{C}_{s}+E_{t, s, u} \mathbf{C}_{u}+F_{t, s, u} \mathbf{I}\right) .
\end{aligned}
$$

Proof of Lemma 3.5. We start from calculating $\mathbb{E}\left[X_{t} p_{n-1}\left(X_{u} ; u\right) \mid \mathcal{F}_{\leq s}\right]$ in two ways. On the one hand,

$$
\begin{aligned}
& \mathbb{E}\left[X_{t} p_{n-1}\left(X_{u} ; u\right) \mid \mathcal{F}_{\leq s}\right]=\mathbb{E}\left[\left(a_{t, s, u} X_{s}+b_{t, s, u} X_{u}\right) p_{n-1}\left(X_{u} ; u\right) \mid \mathcal{F}_{\leq s}\right] \\
& =a_{t, s, u} X_{s} p_{n-1}\left(X_{s} ; s\right)+b_{t, s, u} \mathbb{E}\left[X_{u} p_{n-1}\left(X_{u} ; u\right) \mid \mathcal{F}_{\leq s}\right] \\
& =a_{t, s, u}\left(a_{n-1}(s) p_{n}\left(X_{s} ; s\right)+b_{n-1}(s) p_{n-1}\left(X_{s} ; s\right)+c_{n-1} p_{n-2}\left(X_{s} ; s\right)\right) \\
& +b_{t, s, u}\left(a_{n-1}(u) \mathbb{E}\left[p_{n}\left(X_{u} ; u\right) \mid \mathcal{F}_{\leq s}\right]+b_{n-1}(u) p_{n-1}\left(X_{s} ; s\right)+c_{n-1}(u) p_{n-2}\left(X_{s} ; s\right)\right) .
\end{aligned}
$$

On the other hand,

$$
\begin{aligned}
& \mathbb{E}\left[X_{t} p_{n-1}\left(X_{u} ; u\right) \mid \mathcal{F}_{\leq s}\right] \\
= & \mathbb{E}\left[X_{t} \mathbb{E}\left[p_{n-1}\left(X_{u} ; u\right) \mid \mathcal{F}_{\leq t}\right] \mid \mathcal{F}_{\leq s}\right]=\mathbb{E}\left[X_{t} p_{n-1}\left(X_{t} ; t\right) \mid \mathcal{F}_{\leq s}\right] \\
= & a_{n-1}(t) \mathbb{E}\left[p_{n}\left(X_{t} ; t\right) \mid \mathcal{F}_{\leq s}\right]+b_{n-1}(t) p_{n-1}\left(X_{s} ; s\right)+c_{n-1}(t) p_{n-2}\left(X_{s} ; s\right) .
\end{aligned}
$$


Thus comparing the right hand sides of the above equations we obtain the following equation:

$$
\begin{gathered}
b_{t, s, u} a_{n-1}(u) \mathbb{E}\left[p_{n}\left(X_{u} ; u\right) \mid \mathcal{F}_{\leq s}\right]-a_{n-1}(t) \mathbb{E}\left[p_{n}\left(X_{t} ; t\right) \mid \mathcal{F}_{\leq s}\right] \\
=-p_{n}\left(X_{s} ; s\right) a_{t, s, u} a_{n-1}(s)+p_{n-1}\left(X_{s} ; s\right)\left(b_{n-1}(t)-a_{t, s, u} b_{n-1}(s)-b_{t, s, u} b_{n-1}(u)\right) \\
+p_{n-2}\left(X_{s} ; s\right)\left(c_{n-1}(t)-a_{t, s, u} c_{n-1}(s)-b_{t, s, u} c_{n-1}(u)\right)
\end{gathered}
$$

A trivial verification, using (2.20), shows that

$$
\begin{aligned}
& b_{n-1}(t)-a_{t, s, u} b_{n-1}(s)-b_{t, s, u} b_{n-1}(u)=0, \\
& c_{n-1}(t)-a_{t, s, u} c_{n-1}(s)-b_{t, s, u} c_{n-1}(u)=0 .
\end{aligned}
$$

Hence we have (3.15). To obtain a second equation for $\mathcal{X}$ and $\mathcal{Y}$ let us consider $\mathbb{E}\left[X_{t}^{2} p_{n-2}\left(X_{u} ; u\right) \mid \mathcal{F}_{\leq s}\right]$. On the one hand,

$$
\begin{aligned}
\mathbb{E}\left[X_{t}^{2} p_{n-2}\left(X_{u} ; u\right) \mid \mathcal{F}_{\leq s}\right] & =\mathbb{E}\left[X_{t}^{2} \mathbb{E}\left[p_{n-2}\left(X_{u} ; u\right) \mid \mathcal{F}_{\leq t}\right] \mid \mathcal{F}_{\leq s}\right] \\
& =\mathbb{E}\left[X_{t}^{2} p_{n-2}\left(X_{t} ; t\right) \mid \mathcal{F}_{\leq s}\right]
\end{aligned}
$$

Setting $a_{k}(t)=b_{k}(t)=c_{k}(t)=0$ for $k<0$, a repeated application of (2.19) gives

$$
\begin{aligned}
& \mathbb{E}\left[X_{t}^{2} p_{n-2}\left(X_{u} ; u\right) \mid \mathcal{F}_{\leq s}\right]=a_{n-2}(t) a_{n-1}(t) \mathcal{Y} \\
& \quad+p_{n-1}\left(X_{s} ; s\right) a_{n-2}(t)\left[b_{n-2}(t)+b_{n-1}(t)\right] \\
& \quad+p_{n-2}\left(X_{s} ; s\right)\left[a_{n-2}(t) c_{n-1}(t)+b_{n-2}^{2}(t)+c_{n-2}(t) a_{n-3}(t)\right] \\
& \quad+p_{n-3}\left(X_{s} ; s\right) c_{n-2}(t)\left[b_{n-3}(t)+b_{n-2}(t)\right]+p_{n-4}\left(X_{s} ; s\right) c_{n-3}(t) c_{n-2}(t) .
\end{aligned}
$$

On the other hand, one can rewrite $\mathbb{E}\left[X_{t}^{2} p_{n-2}\left(X_{u} ; u\right) \mid \mathcal{F}_{\leq s}\right]$ as

$$
\begin{gathered}
\mathbb{E}\left[X_{t}^{2} p_{n-2}\left(X_{u} ; u\right) \mid \mathcal{F}_{\leq s}\right]=\mathbb{E}\left[\mathbb{E}\left[X_{t}^{2} \mid \mathcal{F}_{s, u}\right] p_{n-2}\left(X_{u} ; u\right) \mid \mathcal{F}_{\leq s}\right] \\
=A_{t, s, u} X_{s}^{2} p_{n-2}\left(X_{s} ; s\right)+B_{t, s, u} X_{s} \mathbb{E}\left[X_{u} p_{n-2}\left(X_{u} ; u\right) \mid \mathcal{F}_{\leq s}\right] \\
+C_{t, s, u} \mathbb{E}\left[X_{u}^{2} p_{n-2}\left(X_{u} ; u\right) \mid \mathcal{F}_{\leq s}\right]+D_{t, s, u} X_{s} p_{n-2}\left(X_{s} ; s\right) \\
+E_{t, s, u} \mathbb{E}\left[X_{u} p_{n-2}\left(X_{u} ; u\right) \mid \mathcal{F}_{\leq s}\right]+F_{t, s, u} p_{n-2}\left(X_{s} ; s\right)
\end{gathered}
$$

After some algebra one gets

$$
\begin{array}{r}
(\mathrm{A} .21) \quad \mathbb{E}\left[X_{t}^{2} p_{n-2}\left(X_{u} ; u\right) \mid \mathcal{F}_{\leq s}\right]=\mathcal{X} C_{t, s, u} a_{n-2}(u) a_{n-1}(u) \\
+p_{n}\left(X_{s} ; s\right) a_{n-1}(s) R_{n}(t, s, u)+p_{n-1}\left(X_{s} ; s\right) S_{n}(t, s, u)+p_{n-2}\left(X_{s} ; s\right) T_{n}(t, s, u) \\
+p_{n-3}\left(X_{s} ; s\right) U_{n}(t, s, u)+p_{n-4}\left(X_{s} ; s\right) V_{n}(t, s, u)
\end{array}
$$


where

$$
\begin{aligned}
R_{n}(t, s, u)= & A_{t, s, u} a_{n-2}(s)+B_{t, s, u} a_{n-2}(u), \\
S_{n}(t, s, u)= & A_{t, s, u} a_{n-2}(s)\left[b_{n-2}(s)+b_{n-1}(s)\right] \\
& +B_{t, s, u}\left[a_{n-2}(u) b_{n-1}(s)+a_{n-2}(s) b_{n-2}(u)\right] \\
& +C_{t, s, u} a_{n-2}(u)\left[b_{n-2}(u)+b_{n-1}(u)\right]+D_{t, s, u} b_{n-2}(s) \\
& +E_{t, s, u} b_{n-2}(u)+F_{t, s, u}, \\
T_{n}(t, s, u)= & A_{t, s, u}\left[a_{n-2}(s) c_{n-1}(s)+b_{n-2}^{2}(s)+c_{n-2}(s) a_{n-3}(s)\right] \\
& +B_{t, s, u}\left[a_{n-2}(u) c_{n-1}(s)+b_{n-2}(u) b_{n-2}(s)+c_{n-2}(u) a_{n-3}(s)\right] \\
& +C_{t, s, u}\left[a_{n-2}(u) c_{n-1}(u)+b_{n-2}^{2}(u)+c_{n-2}(u) a_{n-3}(u)\right] \\
& +D_{t, s, u} b_{n-2}(s)+E_{t, s, u} b_{n-2}(u)+F_{t, s, u}, \\
U_{n}(t, s, u)= & A_{t, s, u} c_{n-2}(s)\left[b_{n-3}(s)+b_{n-2}(s)\right] \\
& +B_{t, s, u}\left[b_{n-2}(u) c_{n-2}(s)+c_{n-2}(u) b_{n-3}(s)\right] \\
& +C_{t, s, u} c_{n-2}(u)\left[b_{n-3}(u)+b_{n-2}(u)\right]+D_{t, s, u} c_{n-2}(s)+E_{t, s, u} c_{n-2}(u), \\
V_{n}(t, s, u)= & A_{t, s, u} c_{n-2}(s) c_{n-3}(s)+B_{t, s, u} c_{n-2}(u) c_{n-3}(s)+C_{t, s, u} c_{n-2}(u) c_{n-3}(u) .
\end{aligned}
$$

Comparing the right hand side of (A.21) with the right hand side of (A.20) we get the second equation with unknowns $\mathcal{X}$ and $\mathcal{Y}$ :

$$
\begin{array}{r}
C_{t, s, u} a_{n-2}(u) a_{n-1}(u) \mathcal{X}-a_{n-2}(t) a_{n-1}(t) \mathcal{Y}=-p_{n}\left(X_{s} ; s\right) R_{n}(t, s, u) \\
+p_{n-1}\left(X_{s} ; s\right)\left(a_{n-2}(t)\left[b_{n-2}(t)+b_{n-1}(t)\right]-S_{n}(t, s, u)\right) \\
+p_{n-2}\left(X_{s} ; s\right)\left(a_{n-2}(t) c_{n-1}(t)+b_{n-2}^{2}(t)+c_{n-2}(t) a_{n-3}(t)-T_{n}(t, s, u)\right) \\
+p_{n-3}\left(X_{s} ; s\right)\left(c_{n-2}(t)\left[b_{n-3}(t)+b_{n-2}(t)\right]-U_{n}(t, s, u)\right) \\
+p_{n-4}\left(X_{s} ; s\right)\left(c_{n-2}(t) c_{n-3}(t)-V_{n}(t, s, u)\right) .
\end{array}
$$

A calculation based on (2.20) and (3.6)-(3.10) gives

$$
\begin{aligned}
& a_{n-2}(t) {\left[b_{n-2}(t)+b_{n-1}(t)\right]-S_{n}(t, s, u) } \\
&=-\frac{(t-s)(u-t)}{u(1+\sigma s)+\tau-q s}\left[\theta \sigma \alpha_{n-1}+\eta \beta_{n-1}-\beta_{n-1} \gamma_{n-1}+\sigma \tau \alpha_{n-1}\left(\gamma_{n-2}+\gamma_{n-1}\right)\right. \\
&\left.\quad-\sigma \alpha_{n-1} \delta_{n-2}+\sigma \beta_{n-1}\left(\delta_{n-2}+\delta_{n-1}\right)+q\left(\beta_{n-1} \gamma_{n-2}+\sigma \alpha_{n-1} \delta_{n-1}\right)\right], \\
& a_{n-2}(t) c_{n-1}(t)+b_{n-2}^{2}(t)+c_{n-2}(t) a_{n-3}(t)-T_{n}(t, s, u) \\
&=-\frac{(t-s)(u-t)}{u(1+\sigma s)+\tau-q s}\left[1+\theta \gamma_{n-2}+\eta \delta_{n-2}-\gamma_{n-2} \delta_{n-2}-\beta_{n-1} \epsilon_{n-1}\right. \\
& \quad+\tau\left(\gamma_{n-2}^{2}+\sigma \alpha_{n-2} \epsilon_{n-2}+\sigma \alpha_{n-1} \varepsilon_{n-1}\right)-\sigma \alpha_{n-2} \phi_{n-2} \\
&\left.\quad+q\left(\gamma_{n-2} \delta_{n-2}+\beta_{n-2} \epsilon_{n-2}+\sigma \alpha_{n-1} \phi_{n-1}\right)+\sigma\left(\delta_{n-2}^{2}+\beta_{n-2} \phi_{n-2}+\beta_{n-1} \phi_{n-1}\right)\right], \\
& c_{n-2}(t)\left[b_{n-3}(t)+b_{n-2}(t)\right]-U_{n}(t, s, u) \\
&=-\frac{(t-s)(u-t)}{u(1+\sigma s)+\tau-q s}\left[\theta \varepsilon_{n-2}+\tau \varepsilon_{n-2}\left(\gamma_{n-3}+\gamma_{n-2}\right)-\delta_{n-2} \varepsilon_{n-2}+\eta \phi_{n-2}\right. \\
&\left.\quad-\phi_{n-2} \gamma_{n-3}+\sigma\left(\delta_{n-3}+\delta_{n-2}\right) \phi_{n-2}+q\left(\delta_{n-3} \varepsilon_{n-2}+\gamma_{n-2} \phi_{n-2}\right)\right],
\end{aligned}
$$




$$
\begin{aligned}
& c_{n-2}(t) c_{n-3}(t)-V_{n}(t, s, u) \\
& \quad=-\frac{(t-s)(u-t)}{u(1+\sigma s)+\tau-q s}\left[\varepsilon_{n-3}\left(\tau \varepsilon_{n-2}-\phi_{n-2}\right)+\phi_{n-3}\left(q \varepsilon_{n-2}+\sigma \phi_{n-2}\right)\right] .
\end{aligned}
$$

Therefore, by (2.23)-(2.26) the coefficients at $p_{n-1}\left(X_{s} ; s\right), \ldots, p_{n-4}\left(X_{s} ; s\right)$ on the right hand side of (A.22) vanish, and (A.22) is equivalent to (3.16).

Proof of Lemma 3.6, Increasing $\varepsilon$ if necessary, without loss of generality we may assume that $\varepsilon>0$. Let $N(x)=P(|X| \geq x)+P(|Y| \geq x), K=2 / \rho$. Throughout the proof, $C_{1}, C_{2}$ denote positive constants which might differ at each occurrence. The event $\{|X| \geq K x\}$, where $x>0$ is fixed, can be decomposed into the sum of two disjoint events $\{|X| \geq K x\} \cap\{|Y| \geq x\}$ and $\{|X| \geq K x\} \cap\{|Y|<x\}$. Therefore, denoting

$$
\begin{aligned}
& P_{1}(x)=\operatorname{Pr}(|X| \geq x,|Y| \geq x), \\
& P_{2}(x)=\operatorname{Pr}(|X| \geq K x,|Y|<x), \\
& P_{3}(x)=\operatorname{Pr}(|Y| \geq K x,|X|<x),
\end{aligned}
$$

we get $\operatorname{Pr}(|X| \geq K x) \leq P_{1}(x)+P_{2}(x)$, and hence by symmetry of the assumptions,

$$
N(K x) \leq 2 P_{1}(x)+P_{2}(x)+P_{3}(x) .
$$

To estimate $P_{1}(x)$, we observe the following.

Claim A.1. If $|X| \geq x,|Y| \geq x$, and $0<a<1$, then either

$$
(X-\rho Y)^{2} \geq a(1-\rho)^{2} Y^{2}+(1-\rho)^{2}(1-a) x^{2},
$$

or

$$
(Y-\rho X)^{2} \geq a(1-\rho)^{2} X^{2}+(1-\rho)^{2}(1-a) x^{2} .
$$

Indeed, suppose that both inequalities fail; i.e., on the set $\{|X| \geq x,|Y| \geq x\}$ we have $(X-\rho Y)^{2}<a(1-\rho)^{2} X^{2}+(1-\rho)^{2}(1-a) x^{2}$ and $(Y-\rho X)^{2}<a(1-\rho)^{2} Y^{2}+$ $(1-\rho)^{2}(1-a) x^{2}$. Adding the inequalities we obtain

$$
\left(1+\rho^{2}\right)\left(X^{2}+Y^{2}\right)-4 \rho X Y<a(1-\rho)^{2}\left(X^{2}+Y^{2}\right)+2(1-\rho)^{2}(1-a) x^{2} .
$$

Since $4 \rho X Y \leq 2 \rho\left(X^{2}+Y^{2}\right)$, this gives

$$
(1-\rho)^{2}(1-a)\left(X^{2}+Y^{2}\right)<2(1-\rho)^{2}(1-a) x^{2} .
$$

However, since $|X| \geq x,|Y| \geq x$, we have

$$
(1-\rho)^{2}(1-a)\left(X^{2}+Y^{2}\right) \geq 2 x^{2}(1-\rho)^{2}(1-a),
$$

a contradiction.

Claim A.1 with $a=1 / 2$ implies that

$$
\begin{aligned}
& P_{1}(x) \leq \operatorname{Pr}\left(|X-\rho Y| \geq(1-\rho) \sqrt{Y^{2}+x^{2}} / \sqrt{2},|Y| \geq x\right) \\
& \quad+\operatorname{Pr}\left(|Y-\rho X| \geq(1-\rho) \sqrt{X^{2}+x^{2}} / \sqrt{2},|X| \geq x\right) .
\end{aligned}
$$


From the conditional Chebyshev inequality and (3.23) we get

$$
\begin{aligned}
\operatorname{Pr} & \left(|X-\rho Y| \geq(1-\rho) \sqrt{Y^{2}+x^{2}} / \sqrt{2},|Y| \geq x\right) \\
\leq & 2 \int_{|Y| \geq x} \frac{A+B|Y|+\left(1-\rho^{2}\right) \varepsilon^{2} Y^{2} /\left(1+\varepsilon^{2}\right)}{(1-\rho)^{2}\left(Y^{2}+x^{2}\right)} d P \\
\leq & \int_{|Y| \geq x} \frac{2 A}{(1-\rho)^{2} x^{2}} d P+\int_{|Y| \geq x} \frac{2 B|Y|}{(1-\rho)^{2}|Y| x} d P \\
& +\int_{|Y| \geq x} \frac{\left(1-\rho^{2}\right) 2 \varepsilon^{2} Y^{2} /\left(1+\varepsilon^{2}\right)}{(1-\rho)^{2} Y^{2}} d P \\
\leq & \frac{C_{1}}{x^{2}} \operatorname{Pr}(|Y| \geq x)+\frac{C_{2}}{x} \operatorname{Pr}(|Y| \geq x)+\frac{2 \varepsilon^{2}}{1+\varepsilon^{2}} \frac{1+\rho}{1-\rho} \operatorname{Pr}(|Y| \geq x),
\end{aligned}
$$

where $C_{1}=2 A /(1-\rho)^{2}, C_{2}=2 B /(1-\rho)^{2}$. Since the assumptions are symmetric in $X, Y$, this shows that there are constants $C_{1}, C_{2}<\infty$ such that

$$
P_{1}(x) \leq \frac{C_{1} N(x)}{x^{2}}+\frac{C_{2} N(x)}{x}+\frac{2 \varepsilon^{2}}{1+\varepsilon^{2}} \frac{1+\rho}{1-\rho} N(x) .
$$

To estimate $P_{2}(x)$, we use the trivial estimate $|Y-\rho X| \geq \rho|X|-|Y|$, which shows that the event $\{|X| \geq K x,|Y|<x\}$ implies that

$$
|Y-\rho X| \geq \varepsilon|X|+((\rho-\varepsilon) K-1) x=\varepsilon|X|+\frac{\rho-2 \varepsilon}{\rho} x .
$$

Therefore

$$
\begin{aligned}
P_{2}(x) \leq & \operatorname{Pr}\left(|Y-\rho X| \geq \varepsilon|X|+\frac{\rho-2 \varepsilon}{\rho} x,|X| \geq K x\right) \\
= & \int_{|X| \geq K x} \operatorname{Pr}\left(|Y-\rho X| \geq \varepsilon|X|+\frac{\rho-2 \varepsilon}{\rho} x \mid X\right) d P \\
\leq & \int_{|X| \geq K x} \frac{A+B|X|+\left(1-\rho^{2}\right) \varepsilon^{2} X^{2} /\left(1+\varepsilon^{2}\right)}{(\varepsilon|X|+(\rho-2 \varepsilon) x / \rho)^{2}} d P \\
\leq & \int_{|X| \geq K x} \frac{A}{((\rho-2 \varepsilon) x / \rho)^{2}} d P+\int_{|X| \geq K x} \frac{B|X|}{\varepsilon|X|(\rho-2 \varepsilon) x / \rho} d P \\
& +\int_{|X| \geq K x} \frac{\left(1-\rho^{2}\right) \varepsilon^{2} X^{2}}{\left(1+\varepsilon^{2}\right) \varepsilon^{2} X^{2}} d P .
\end{aligned}
$$

Since $N(K x) \leq N(x)$, this shows that

$$
P_{2}(x) \leq \frac{C_{1} N(x)}{x^{2}}+\frac{C_{2} N(x)}{x}+\frac{1-\rho^{2}}{1+\varepsilon^{2}} \operatorname{Pr}(|X| \geq K x),
$$

where $C_{1}=\rho^{2} A /(\rho-2 \varepsilon)^{2}, C_{2}=\rho B /(\varepsilon(\rho-2 \varepsilon))$. By symmetry of the assumptions, we also have

$$
P_{3}(x) \leq \frac{C_{1} N(x)}{x^{2}}+\frac{C_{2} N(x)}{x}+\frac{1-\rho^{2}}{1+\varepsilon^{2}} \operatorname{Pr}(|Y| \geq K x) .
$$

Combining (A.23), (A.24), A.25), and (A.26) we obtain that there are constants $C_{3}, C_{4}>0$ such that

$$
N(K x) \leq \frac{C_{3} N(x)}{x^{2}}+\frac{C_{4} N(x)}{x}+\frac{1-\rho^{2}}{1+\varepsilon^{2}} N(K x)+\frac{4 \varepsilon^{2}}{1+\varepsilon^{2}} \frac{1+\rho}{1-\rho} N(x),
$$

which implies (3.25). 
Proof of Lemma 3.26. Clearly, (3.26) implies that $2 \varepsilon<\rho$. Indeed,

$$
2^{2} \varepsilon^{2}<2^{p+3} \varepsilon^{2} \frac{1+\rho}{1-\rho} \leq \rho^{p+3}<\rho^{2} .
$$

We use Lemma 3.6 and we use the notation $N(x)=P(|X| \geq x)+P(|Y| \geq x)$, $K=2 / \rho$ introduced in its proof. Fix $M>0$. Then, noticing that $K>1$, from Lemma 3.6 we get

$$
\begin{gathered}
\int_{0}^{M}(p+1) x^{p} N(x) d x=K^{p+1} \int_{0}^{M / K}(p+1) x^{p} N(K x) d x \\
\leq \frac{p+1}{p-1} C_{1} \int_{0}^{\infty}(p-1) x^{p-2} N(x) d x+\frac{p+1}{p} C_{2} \int_{0}^{\infty} p x^{p-1} N(x) d x \\
\quad+\frac{4 \varepsilon^{2}}{\rho^{2}+\varepsilon^{2}} \frac{1+\rho}{1-\rho} K^{p+1} \int_{0}^{M}(p+1) x^{p} N(x) d x \\
<\frac{p+1}{p-1} C_{1}\left(\mathbb{E}\left(|X|^{p-1}\right)+\mathbb{E}\left(|Y|^{p-1}\right)\right)+\frac{p+1}{p} C_{2}\left(\mathbb{E}\left(|X|^{p}\right)+\mathbb{E}\left(|Y|^{p}\right)\right) \\
+\varepsilon^{2} 2^{p+3} / \rho^{p+3} \frac{1+\rho}{1-\rho} \int_{0}^{M}(p+1) x^{p} N(x) d x .
\end{gathered}
$$

Therefore, if (3.26) holds true, then $\sup _{M>0} \int_{0}^{M}(p+1) x^{p} N(x) d x<\infty$, which implies $\mathbb{E}\left(|X|^{p+1}\right)+\mathbb{E}\left(|Y|^{p+1}\right)=\int_{0}^{\infty}(p+1) x^{p} N(x) d x<\infty$.

\section{ACKNOWLEDGEMENT}

We would like to thank M. Bożejko for numerous discussions and hospitality, L. Gallardo for a preprint of 22], M. Ismail, D. Pommeret, J. Wysoczański, M. Yor and V. Zarikian for helpful discussions. The second and the third authors are very grateful to their hosts for providing excellent research facilities and friendly atmosphere during their visit to the Department of Mathematics, University of Cincinnati, in August and September 2004.

\section{REFERENCES}

[1] W. A. Al-Salam and T. S. Chihara. Convolutions of orthonormal polynomials. SIAM J. Math. Anal., 7(1):16-28, 1976. MR0399537 (53:3381)

[2] Michael Anshelevich. Free martingale polynomials. Journal of Functional Analysis, (201):228-261, 2003. arXiv:math.CO/0112194. MR1986160 (2004f:46079)

[3] Nobuhiro Asai, Izumi Kubo, and Hui-Hsiung Kuo. Segal-Bargmann transforms of one-mode interacting Fock spaces associated with Gaussian and Poisson measures. Proc. Amer. Math. Soc., 131(3):815-823, 2003. MR1937419 (2003j:46099)

[4] Richard Askey and Mourad Ismail. Recurrence relations, continued fractions, and orthogonal polynomials. Mem. Amer. Math. Soc., 49(300):iv+108, 1984. MR743545 (85g:33008)

[5] Richard Askey and James Wilson. Some basic hypergeometric orthogonal polynomials that generalize Jacobi polynomials. Mem. Amer. Math. Soc., 54(319):iv+55, 1985. MR783216 (87a:05023)

[6] Marek Bożejko and Włodzimierz Bryc. On a class of free Lévy laws related to a regression problem. J. Funct. Anal. 236:59-77, 2006. MR2227129 (2007a:46071)

[7] Marek Bożejko, Burkhard Kümmerer, and Roland Speicher. q-Gaussian processes: noncommutative and classical aspects. Comm. Math. Phys., 185(1):129-154, 1997. MR 1463036 (98h:81053)

[8] Marek Bożejko and Janusz Wysoczański. Remarks on $t$-transformations of measures and convolutions. Ann. Inst. H. Poincaré Probab. Statist., 37(6):737-761, 2001. MR $\overline{1863276}$ (2002i:60005) 
[9] Włodzimierz Bryc. Some remarks on random vectors with nice enough behaviour of conditional moments. Bull. Polish Acad. Sci., 33:677-683, 1985. MR849420 (88d:60021)

[10] Włodzimierz Bryc, Wojciech Matysiak, and Jacek Wesołowski. The bi-Poisson process: A quadratic harness. Ann. Probab., to appear.

[11] Włodzimierz Bryc and Agnieszka Plucińska. A characterization of infinite Gaussian sequences by conditional moments. Sankhya A, 47:166-173, 1985. MR844017 (87k:62023)]

[12] Włodzimierz Bryc and Jacek Wesołowski. Bi-Poisson process. Infin. Dimens. Anal. Quantum Probab. Related. Top., to appear.

[13] Włodzimierz Bryc and Jacek Wesołowski. The classical bi-Poisson process: An invertible quadratic harness. Statist. Probab. Lett. 76:1664-1674, 2006. MR2248855

[14] Włodzimierz Bryc and Jacek Wesołowski. Conditional moments of $q$-Meixner processes. Probability Theory Related Fields, 131:415-441, 2005. arXiv:math.PR/0403016. MR2123251 (2005k:60233)

[15] T. S. Chihara. An introduction to orthogonal polynomials. Gordon and Breach, New York, 1978. MR0481884 (58:1979)

[16] B. Derrida, M. R. Evans, V. Hakim, and V. Pasquier. Exact solution of a 1D asymmetric exclusion model using a matrix formulation. J. Phys. A, 26(7):1493-1517, 1993. MR 1219679 (94g:60179)

[17] M. Dozzi. Two-parameter harnesses and the Wiener process. Z. Wahrsch. Verw. Gebiete, 56(4):507-514, 1981. MR621661 (82h:60152)

[18] Charles F. Dunkl and Yuan Xu. Orthogonal polynomials of several variables, volume 81 of Encyclopedia of Mathematics and its Applications. Cambridge University Press, Cambridge, 2001. MR1827871 (2002m:33001)

[19] Fabian H. L. Essler and Vladimir Rittenberg. Representations of the quadratic algebra and partially asymmetric diffusion with open boundaries. J. Phys. A, 29(13):3375-3407, 1996. MR:1400161 (97b:82017)

[20] Philip Feinsilver. Lie algebras and recurrence relations. III. $q$-analogs and quantized algebras. Acta Appl. Math., 19(3):207-251, 1990. MR.1077860(93k:33012)

[21] U. Frisch and R. Bourret. Parastochastics. J. Math. Phys., 11(2):364-390, 1970. MR0260352 $(41: 4979)$

[22] Léonard Gallardo and Marc Yor. Some new examples of Markov processes which enjoy the time-inversion property. Probab. Theory Related Fields, 132(1):150-162, 2005. MR 2136870 (2006e:60106)

[23] J. M. Hammersley. Harnesses. In Proc. Fifth Berkeley Sympos. Mathematical Statistics and Probability (Berkeley, California, 1965/66), Vol. III: Physical Sciences, pages 89-117. Univ. California Press, Berkeley, California, 1967. MR0224144 (36:7190)

[24] Mourad E. H. Ismail and Dennis Stanton. $q$-integral and moment representations for $q$ orthogonal polynomials. Canad. J. Math., 54(4):709-735, 2002. MR1913916 (2003k:33024)

[25] Christian Kassel. Quantum groups, volume 155 of Graduate Texts in Mathematics. SpringerVerlag, New York, 1995. MR1321145 (96e:17041)

[26] Anna Krystek and Lukasz Wojakowski. Associative convolutions arising from conditionally free convolution. Infinite Dimensional Analysis, Quantum Probability and Related Topics, 8:515-545, 2005. MR:2172313 (2006g:46103)

[27] I. G. Macdonald. Affine Hecke algebras and orthogonal polynomials, volume 157 of Cambridge Tracts in Mathematics. Cambridge University Press, Cambridge, 2003. MR1976581 (2005b:33021)

[28] R. Mansuy and M. Yor. Harnesses, Lévy bridges and Monsieur Jourdain. Stochastic Processes and Their Applications, 115:329-338, February 2005. MR2111197(2005m:60104)

[29] Masatoshi Noumi and Jasper V. Stokman. Askey-Wilson polynomials: An affine Hecke algebra approach. In Laredo Lectures on Orthogonal Polynomials and Special Functions, Adv. Theory Spec. Funct. Orthogonal Polynomials, pages 111-144. Nova Sci. Publ., Hauppauge, New York, 2004. MR2085854 (2005h:42057)

[30] Eugene A. Pechersky Pablo A. Ferrari, and Beat M. Niederhauser. Harness processes and non-homogeneous crystals. arXiv:math.PR/0409301, 2004.

[31] A. Perelomov. Generalized coherent states and their applications. Texts and Monographs in Physics. Springer-Verlag, Berlin, 1986. MR858831 (87m:22035)

[32] André Ronveaux and Walter Van Assche. Upward extension of the Jacobi matrix for orthogonal polynomials. J. Approx. Theory, 86(3):335-357, 1996. MR1405986 (97k:42054) 
[33] Gian-Carlo Rota. Finite Operator Calculus. Academic Press, 1975. MR0379213 (52:119)

[34] Gabriela Sansigre and Galliano Valent. A large family of semi-classical polynomials: The perturbed Chebyshev. J. Comput. Appl. Math., 57(1-2):271-281, 1995. MR1340942 (96f:33021)

[35] Wim Schoutens. Stochastic processes and orthogonal polynomials, volume 146 of Lecture Notes in Statistics. Springer-Verlag, New York, 2000. MR.1761401(2001f:60095)

[36] Masaru Uchiyama, Tomohiro Sasamoto, and Miki Wadati. Asymmetric simple exclusion process with open boundaries and Askey-Wilson polynomials. J. Phys. A, 37(18):4985-5002, 2004. MR2065218 (2006d:82047)

[37] Hans van Leeuwen and Hans Maassen. A $q$-deformation of the Gauss distribution. J. Math. Phys., 36(9):4743-4756, 1995. MR 1347109 (97a:81104)

[38] A. M. Vershik. Algebras with quadratic relations. Selecta Math. Soviet., 11(4):293-315, 1992. Selected translations. MR 1206295

[39] Jacek Wesołowski. Stochastic processes with linear conditional expectation and quadratic conditional variance. Probab. Math. Statist., 14:33-44, 1993. MR.1267516 (95b:60061)

[40] David Williams. Some basic theorems on harnesses. In Stochastic analysis (a tribute to the memory of Rollo Davidson), pages 349-363. Wiley, London, 1973. MR0362565 (50:15005)

[41] Zhan Gong Zhou. Two-parameter harnesses and the generalized Brownian sheet. Natur. Sci. J. Xiangtan Univ., 14(2):111-115, 1992. MR.1184355(93h:60129)

[42] Xing Wu Zhuang. The generalized Brownian sheet and two-parameter harnesses. Fujian Shifan Daxue Xuebao Ziran Kexue Ban, 4(4):1-9, 1988. MR1041927(91e:60158)

Department of Mathematics, University of Cincinnati, P.O. Box 210025, Cincinnati, Оніо 45221-0025

E-mail address: Wlodzimierz.Bryc@UC.edu

Faculty of Mathematics and Information Science, Warsaw University of Technology, Pl. Politechniki 1, 00-661 Warszawa, Poland

E-mail address: matysiak@mini.pw.edu.pl

Faculty of Mathematics and Information Science, Warsaw University of Technology, Pl. Politechniki 1, 00-661 Warszawa, Poland

E-mail address: wesolo@alpha.mini.pw.edu.pl 